Groups Geom. Dyn. 8 (2014), 39-68

DOI $10.4171 / \mathrm{GGD} / 216$
Groups, Geometry, and Dynamics

(C) European Mathematical Society

\title{
Automorphisms of curve complexes on nonorientable surfaces
}

\author{
Ferihe Atalan and Mustafa Korkmaz
}

\begin{abstract}
For a compact connected nonorientable surface $N$ of genus $g$ with $n$ boundary components, we prove that the natural map from the mapping class group of $N$ to the automorphism group of the curve complex of $N$ is an isomorphism provided that $g+n \geq 5$. We also prove that two curve complexes are isomorphic if and only if the underlying surfaces are diffeomorphic.
\end{abstract}

Mathematics Subject Classification (2010). 57M60, $20 \mathrm{~F} 38$.

Keywords. Mapping class group, complex of curves, nonorientable surface.

\section{Introduction and statement of results}

1.1. The results. Let $N$ be a compact connected nonorientable surface of genus $g$ with $n$ holes (= boundary components) and let $\operatorname{Mod}(N)$ denote the mapping class group of $N$, the group of isotopy classes of all diffeomorphisms $N \rightarrow N$. The $\operatorname{group} \operatorname{Mod}(N)$ acts on the curve complex $C(N)$ on $N$ (defined in Section 2) as simplicial automorphisms. In other words, there is a natural group homomorphism $\operatorname{Mod}(N) \rightarrow$ Aut $C(N)$. The main result of this paper is the following theorem.

Theorem 1. Let $g+n \geq 5$ and let $N$ be a compact connected nonorientable surface of genus $g$ with $n$ holes. Then the natural map $\operatorname{Mod}(N) \rightarrow \operatorname{Aut} C(N)$ is an isomorphism.

Our second result is the classification of curve complexes on all surfaces, including orientable surfaces. This is given below, and also stated as Theorem 4.6 in Section 4.

Theorem 2. Let $\Sigma_{1}$ and $\Sigma_{2}$ be two compact connected surfaces such that the curve complexes $C\left(\Sigma_{1}\right)$ and $C\left(\Sigma_{2}\right)$ are nonempty and $\left(\Sigma_{1}, \Sigma_{2}\right)$ is not an exceptional pair (see Section 4 for exceptional pairs). Then the simplicial complexes $C\left(\Sigma_{1}\right)$ and $C\left(\Sigma_{2}\right)$ are isomorphic if and only if the surfaces $\Sigma_{1}$ and $\Sigma_{2}$ are diffeomorphic. 
1.2. History. The complex of curves on an orientable surface $S$ was introduced by Harvey [4]. It was shown by Ivanov [11] that if the genus of $S$ is at least two, then the natural map $\operatorname{Mod}^{*}(S) \rightarrow$ Aut $C(S)$ is an isomorphism, with the exception of a closed surface of genus two, in which case this map is onto and its kernel is the cyclic subgroup generated by the hyperelliptic involution. Here, $\operatorname{Mod}^{*}(S)$ denotes the extended mapping class group, the group of isotopy classes of all selfdiffeomorphisms of $S$. This result was extended to lower genus cases by the second author [13], and also by Luo [15], who gave another proof in all cases. Theorem 1 extends this result to nonorientable surfaces.

Theorem 3 ([11], [13], [15]). Let $S$ be a compact connected orientable surface of genus $g$ with $n$ holes. Suppose that $S$ is neither a sphere with at most four holes, nor a torus with at most two holes, nor a closed surface of genus two. Then the natural map $\operatorname{Mod}^{*}(S) \rightarrow$ Aut $C(S)$ is an isomorphism. If $S$ is a closed surface of genus two, then this map is onto and the kernel is the subgroup of order two generated by the hyperelliptic involution.

Using this result, Ivanov proved that any isomorphism between two subgroups of finite index in $\operatorname{Mod}^{*}(S)$ is the restriction of an inner automorphism of $\operatorname{Mod}^{*}(S)$, concluding the outer automorphism group of a subgroup of finite index in $\operatorname{Mod}^{*}(S)$ is finite (cf. [11], [13]). It is very likely that Theorem 1, or a version of it might be used to prove the nonorientable version of this result. We hope to do this in a future project.

As an another application, Ivanov [12] gave another proof of a theorem of RoydenEarl-Kra: All isometries of the Teichmüller space are induced by diffeomorphisms of $S$. Ivanov [11] also gave another proof of the fact that the mapping class groups are not arithmetic by using this theorem.

After the appearance of [11], [13] and [15], there have been many analogous results: Schaller [20] studied the graph whose vertices are nonseparating simple closed geodesics and whose edges are pairs of vertices intersecting once, Margalit [16] the pants complex, Brendle-Margalit [3] the complex of separating simple closed curves, Irmak-Korkmaz [9] the Hatcher-Thurston complex. In all these cases, the natural action of the extended mapping class group induces an isomorphism to the automorphism group of the complex. Irmak [5], [6], [7] showed that a superinjective simplicial map of the complex of curves or of the complex of nonseparating curves is induced by a diffeomorphism of the orientable surface.

This paper gives the first such result to study the mapping class groups of nonorientable surfaces. Recently in [8], Irmak used some of our results in this paper to study superinjective maps of the curve complex on a nonorientable surface.

1.3. Idea of proof of Theorem 1. The injectivity of the natural map in Theorem 1 is easy; it follows from Theorem 3. The surjectivity, however, is the harder part, as 
in the proof of Theorem 3, and it occupies most of this article. The proof of it does not follow from Theorem 3 right away, but we use it.

Our proof of surjectivity is by induction on the genus of the surface: We cut the surface along a one-sided simple closed curve to decrease the genus by one and use the connectedness of a graph whose vertices are one-sided simple closed curves. For our purpose, it is convenient to consider a sphere as a nonorientable surface of genus zero, so that the initial step of the induction holds by Theorem 3 . The cutting argument was first used by Ivanov in [11] to obtain his theorem for surfaces of at least two boundary components from surfaces having at most one boundary component. It has been used since then in many many instances. See, for example, [11], [21] and [2].

In her $\mathrm{PhD}$ thesis [1] under the guidance of the second author, the first author proved Theorem 1 for odd genus nonorientable surfaces, proving it first for surfaces of genus one and then by using the cutting argument along two-sided simple closed curves, which decreases the genus by two.

1.4. Outline of the paper. We now give an outline of the paper. In Section 2, we give the necessary definitions and the preliminary information. In Section 3, we define two graphs $X(N)$ and $\tilde{X}(N)$ whose vertices are isotopy classes of one-sided essential (defined below) simple closed curves and whose edges are pairs of dual vertices satisfying certain properties. We investigate the connectedness property of these graphs in Theorem 3.9 and Theorem 3.10. In Section 4, we prove Theorem 2 (= Theorem 4.6) which states that, except for a few sporadic cases, two surfaces (orientable or nonorientable) are diffeomorphic if and only if the corresponding curve complexes are isomorphic. Section 5 shows that automorphisms of the curve complex preserve the topological types of vertices. In Section 6, we prove the injectivity of the map in Theorem 1. This is stated as Theorem 6.1. Section 7 is devoted to the proof of Theorem 7.7, which proves the surjectivity of the map in Theorem 1 . The proof is by induction on the genus of the surface. In Section 8, we treat the cases not covered by Theorem 1 .

Acknowledgment. The authors would like to thank the referee for making several valuable suggestions and corrections.

\section{Basics of the curve complex}

Let $N$ be a compact connected nonorientable surface of genus $g$ with $n$ holes. That is, $N$ is a closed nonorientable surface of genus $g$ from which the interiors of $n$ disjoint disks are removed. Recall that the genus of a nonorientable surface is the number of projective planes in a connected sum decomposition. Equivalently, it is the maximum number of disjoint simple closed curves in $N$ whose complement is connected. (This definition also holds for orientable surfaces.) 
2.1. Circles. A circle $a$ on $N$ is an unoriented simple closed curve. A circle is called two-sided (respectively, one-sided) if a regular neighborhood of it is an annulus (respectively, a Möbius strip). A circle is called trivial if it bounds either a disk, or a disk with one hole, or a Möbius band. A nontrivial circle is a circle which is not trivial.

If $a$ is a circle, then we denote by $N_{a}$ the surface obtained by cutting $N$ along $a$. A circle $a$ is nonseparating if $N_{a}$ is connected and separating otherwise. A one-sided circle $a$ is essential if either $g=1$, or $g \geq 2$ and the surface $N_{a}$ is nonorientable. We denote circles by the lowercase letters $a, b, c$ and their isotopy classes by $\alpha, \beta, \gamma$.

Let $\alpha$ be the isotopy class of a circle $a$. We say that $\alpha$ is nonseparating if $a$ is nonseparating. Similarly for separating, one-sided, two-sided or essential circles.

The geometric intersection number $i(\alpha, \beta)$ of two isotopy classes $\alpha$ and $\beta$ is defined to be the minimum of the cardinality $|a \cap b|$ of $a \cap b$ with $a \in \alpha, b \in \beta$.

2.2. Abstract simplicial complex. An abstract simplicial complex is defined as follows (cf. [17]): Let $V$ be a nonempty set. An abstract simplicial complex $K$ with vertices $V$ is a collection of nonempty finite subsets of $V$, called simplices of $K$, such that

- if $v \in V$, then $\{v\} \in K$, and

- if $\sigma \in K$ and $\tau \subset \sigma$ is nonempty, then $\tau \in K$.

The dimension $\operatorname{dim} \sigma$ of a simplex $\sigma$ is $|\sigma|-1$, where $|\sigma|$ is the cardinality of $\sigma$. A $q$-simplex is a simplex of dimension $q$. The supremum of the dimensions of all simplices of $K$ is the dimension of $K$.

A subcomplex $L$ of an abstract simplicial complex $K$ is called a full subcomplex if whenever a set of vertices of $L$ is a simplex in $K$, it is also a simplex in $L$.

If $X_{1}$ and $X_{2}$ are two abstract simplicial complexes, then the join of $X_{1}$ and $X_{2}$ is the abstract simplicial complex $X_{1} \star X_{2}$ defined as follows: A simplex of $X_{1} \star X_{2}$ is either a simplex of $X_{1}$, or a simplex of $X_{2}$, or the union of a simplex of $X_{1}$ and a simplex of $X_{2}$.

2.3. The curve complex. The complex of curves $C(S)$ on an orientable surface $S$ is the abstract simplicial complex whose simplices are sets of isotopy classes of nontrivial circles which can be represented by pairwise disjoint curves. The complex of curves $C(N)$ on a nonorientable surface $N$ is defined similarly; it is the abstract simplicial complex whose vertices are the isotopy classes of nontrivial circles, and a set of vertices $\left\{\alpha_{0}, \alpha_{1}, \ldots, \alpha_{q}\right\}$ is declared to be a $q$-simplex if and only if $\alpha_{0}, \alpha_{1}, \ldots, \alpha_{q}$ can be represented by pairwise disjoint curves, i.e., $i\left(\alpha_{j}, \alpha_{k}\right)=0$ for all $0 \leq j, k \leq q$ $(j \neq k)$. In particular, two distinct vertices $\alpha$ and $\beta$ of $C(N)$ are joined by an edge if and only if their geometric intersection number is zero.

2.3.1. Dimension of the curve complex. The dimension of $C(N)$ is 0 if $N$ is the projective plane with at most one hole. It is equal to 1 if $N$ is a (closed) Klein bottle. 
If the Euler characteristic of the nonorientable surface $N$ of genus $g$ with $n$ holes is negative, then a simple Euler characteristic argument shows that the maximal number of disjoint nontrivial nonparallel circles on $N$ is $2 g+n-3$. Hence, the dimension of $C(N)$ is $2 g+n-4$.

If $S$ is orientable of genus $g$ with $n$ holes with negative Euler characteristic and if $S$ is not a sphere with three holes, then the dimension of $C(S)$ is $3 g+n-4$. If $S$ is a torus, then the dimension of $C(S)$ is 0 . In the remaining cases, the complex $C(S)$ is empty.

2.3.2. Star, dual and link of a vertex. Let $\alpha$ be a vertex in $C(N)$. The $\operatorname{star} \operatorname{St}(\alpha)$ of $\alpha$ is defined to be the subcomplex of $C(N)$ consisting of all simplices in $C(N)$ containing $\alpha$ and all faces of such simplices. The link $L(\alpha)$ of $\alpha$ is the full subcomplex of $C(N)$ whose simplices are those simplices of $\operatorname{St}(\alpha)$ which do not contain $\alpha$.

Two distinct vertices $\alpha$ and $\beta$ are called $d u a l$ if they have representatives intersecting transversally at one point, i.e., $i(\alpha, \beta)=1$. For a vertex $\alpha$ represented by a one-sided circle, we define the dual $D(\alpha)$ of $\alpha$ to be the set consisting of vertices of $C(N)$ which are dual to $\alpha$.

2.4. Curve complexes in low dimensions. If $S$ is a sphere with $n \leq 3$ holes, then there are no nontrivial circles on $S$, hence, $C(S)$ is empty. If $S$ is a sphere with four holes, or a torus with at most one hole, then $C(S)$ is infinite discrete.

If $N$ is a projective plane with at most one hole, then $C(N)$ consists of one vertex. If $N$ is a projective plane with two holes, then $C(N)$ consists two vertices, the isotopy classes of the circles $c_{1}$ and $c_{2}$ (cf. Figure 1 (i)). If $N$ is a (closed) Klein bottle, then $C(N)$ has three vertices and one edge between two of these vertices. The vertices are the isotopy classes of two one-sided circles, which are connected by the unique edge, and the unique two-sided circle (cf. Figure 1 (ii)).

If $N$ is a Klein bottle with one hole, then $C(N)$ has two connected components, one of which consists of a vertex, the isotopy class of the unique two-sided circle $a$. The other component is a regular tree with countably infinite vertices where each vertex has valence two (cf. [19]).

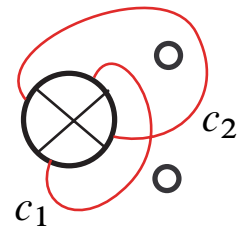

(i)

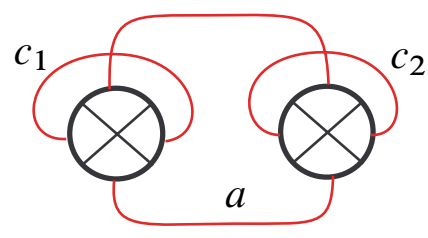

(ii)

Figure 1. The nontrivial circles (up to isotopy) on a real projective plane with two holes, and on a Klein bottle. Here, the interiors of the disks with a cross inside are to be removed and the antipodal points on each resulting boundary component are to be identified. 
2.5. Maximal simplices in the curve complex. We note that a simplex in an abstract simplicial complex is maximal if it is not the proper face of any other simplex. We recall that the maximum number of disjoint pairwise nonisotopic nontrivial circles on a connected orientable surface $S$ of genus $g$ with $n$ boundary components is $3 g+n-3$, whenever the Euler characteristic of $S$ is negative.

Lemma 2.1. Let $S$ be a connected orientable surface of genus $g$ with $n$ holes and with negative Euler characteristic. If $g=0$ suppose, in addition, that $n \geq 4$. Then all maximal simplices in $C(S)$ have the same dimension $3 g+n-4$.

For nonorientable surfaces, however, the situation is quite different.

Lemma 2.2. Let $N$ be a real projective plane with $n \geq 2$ holes. All maximal simplices in $C(N)$ have the same dimension $n-2$.

Proof. Let $n=2$. The complex $C(N)$ consists of only two points. Hence, all simplices are of dimension 0 .

Let $n \geq 3$ and let $\sigma$ be a maximal simplex. Suppose that the dimension of $\sigma$ is dimension $q$, so that $\sigma$ contains $q+1$ elements. Since the genus of the surface is one, there is exactly one one-sided vertex, say $\alpha$, contained in $\sigma$. By cutting $N$ along $a$ for circle $a$ representing $\alpha$, we get the surface $N_{a}$, a sphere with $n+1$ holes. Observe that $\sigma-\{\alpha\}$ gives a maximal simplex in the curve complex $C\left(N_{a}\right)$ of $N_{a}$. By Lemma 2.1, the dimension of $\sigma-\{\alpha\}$ is $n-3$. Thus, $q-1=n-3$, concluding that the dimension of $\sigma$ is $q=n-2$.

Proposition 2.3. Let $N$ be a connected nonorientable surface of genus $g \geq 2$ with $n$ holes. Suppose that $(g, n) \neq(2,0)$. Let $a_{r}=3 r+n-2$ and $b_{r}=4 r+n-2$ if $g=2 r+1$, and $a_{r}=3 r+n-4$ and $b_{r}=4 r+n-4$ if $g=2 r$. Then there is $a$ maximal simplex of dimension $q$ in $C(N)$ if and only if $a_{r} \leq q \leq b_{r}$. In particular, there are precisely $\left\lceil\frac{g}{2}\right\rceil$ values which occur as the dimension of a maximal simplex, where $\left\lceil\frac{g}{2}\right\rceil$ denotes the smallest integer bigger than $\frac{g}{2}$.

Proof. Let $q$ be an integer with $a_{r} \leq q \leq b_{r}$. Write $q=a_{r}+s$, so that $0 \leq s \leq r$. A maximal simplex of dimension $q$ in $C(N)$ is shown in Figure 2.

We now prove the converse. Let $\sigma$ be a maximal simplex of dimension $q$. Hence, $\sigma$ contains $q+1$ elements. Choose pairwise disjoint circles representing the elements of $\sigma$, and let $N_{\sigma}$ denote the surface obtained by cutting $N$ along these circles. Since $\sigma$ is maximal, each connected component of $N_{\sigma}$ is a sphere with three holes (a pair of pants).

Suppose that the number of one-sided vertices in $\sigma$ is $m$, so that there are $q+1-m$ two-sided vertices in $\sigma$. We note that $1 \leq m \leq g$ if $g$ is odd and $0 \leq m \leq g$ if $g$ is even. Let $k$ denote the number of components of $N_{\sigma}$. Since the Euler characteristic of each component is -1 , we see that

$$
k=-\chi(N)=g+n-2,
$$



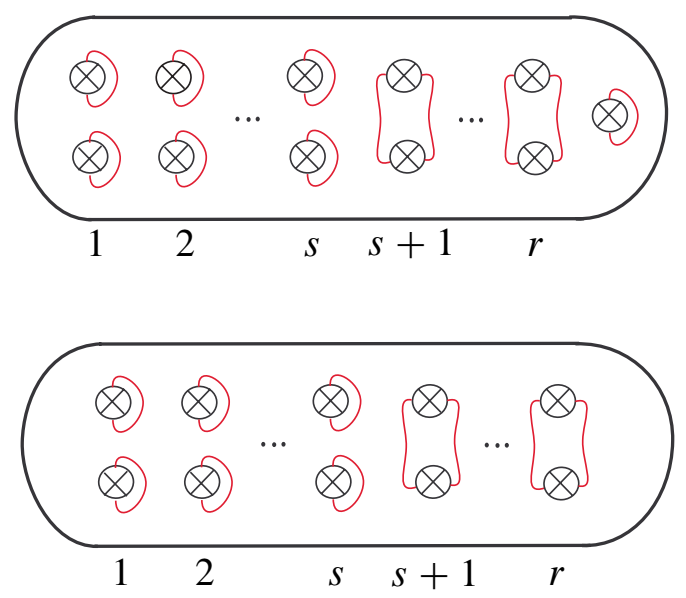

Figure 2. These curves together with any $g+n-3$ pairwise disjoint curves on the complement of them give a maximal simplex of dimension $q=a_{r}+s$. The holes are not drawn.

where $\chi(N)$ is the Euler characteristic of $N$. The number of boundary components of $N_{\sigma}$ is $3 k$. On the other hand, when we cut $N$ along the representatives of $\sigma$, each one-sided circle gives rise to one boundary component and each two-sided circle gives rise to two boundary components in $N_{\sigma}$. It follows that

$$
3 k=m+2(q+1-m)+n=3(g+n-2) .
$$

We see from this that

$$
q=\frac{3 g+m}{2}+n-4
$$

If $g=2 r+1$ then $q=3 r+n-2+\frac{m-1}{2}$, where $1 \leq m \leq 2 r+1$ or $0 \leq \frac{m-1}{2} \leq r$. If $g=2 r$ then $q=3 r+n-4+\frac{m}{2}$, where $0 \leq m \leq 2 r$ or $0 \leq \frac{m}{2} \leq r$.

The proposition follows.

2.6. Mapping class group of a surface of genus one with two holes. Let $N$ denote the nonorientable surface of genus one with two boundary components $\partial_{1}$ and $\partial_{2}$, as shown in Figure 3. In the figure, a disk is glued along the boundary component $d$. For $i=1,2$, choose an oriented $\operatorname{arc} l_{i}$ whose endpoints lie on $\partial_{i}$ as in Figure 3. Sliding $\partial_{i}$ along $l_{i}$ once gives a diffeomorphism of $N$, called a boundary slide, whose isotopy class in $\operatorname{Mod}(N)$ is denoted by $v_{i}$. Note that $v_{i}$ reverses the orientation of $\partial_{i}$.

Let $\operatorname{PMod}(N)$ be the pure mapping class group of $N$, the subgroup of $\operatorname{Mod}(N)$ consisting of isotopy classes of diffeomorphisms mapping each boundary component to itself. Note that $v_{1}$ and $v_{2}$ are in $\operatorname{PMod}(N)$. The next theorem follows from the proof of Corollary 4.6 in [14]. 

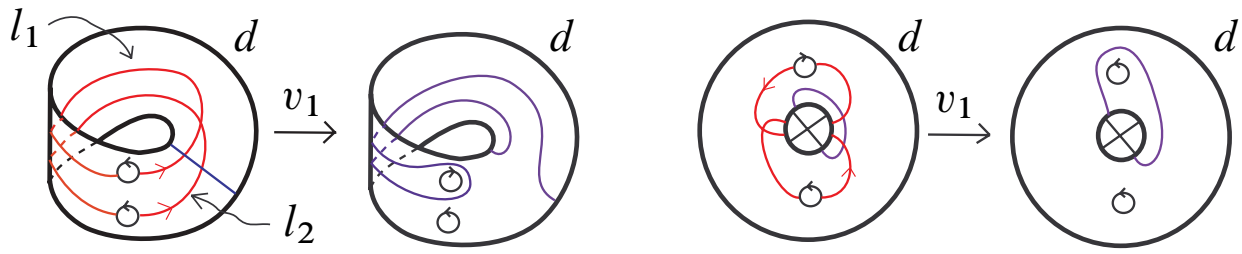

Figure 3. Two representations of the boundary slide $v_{1}$.

Theorem 2.4 ([14]). Let $N$ be a real projective plane with two boundary components $\partial_{1}$ and $\partial_{2}$. The kernel of the natural homomorphism $\operatorname{PMod}(N) \rightarrow \operatorname{Aut} C(N)$ is $\left\{1, v_{1} v_{2}\right\}$, where 1 denotes the isotopy class of the identity.

\section{Two graphs on curves}

Let $N$ be a nonorientable surface of genus $g$ with $n$ holes. We recall that a one-sided circle $a$ is essential if either $g=1$, or $g \geq 2$ and the surface $N_{a}$ obtained by cutting $N$ along $a$ is nonorientable. Note that if $g$ is even, then all one-sided circles on $N$ are essential.

We define a graph $X(N)$ as follows: The vertices of $X(N)$ are the isotopy classes of one-sided essential circles on $N$. We connect two distinct vertices $\alpha$ and $\beta$ in $X(N)$ by an edge if $\alpha$ and $\beta$ are dual, i.e., they have representatives intersecting transversely at one point.

We define a subgraph $\tilde{X}(N)$ of $X(N)$ as follows: The vertices of $\tilde{X}(N)$ are those of $X(N)$. Two distinct vertices $\alpha$ and $\beta$ are connected by an edge in $\tilde{X}(N)$ if $\alpha$ and $\beta$ have representatives $a$ and $b$ intersecting transversely at one point such that

- either $g \geq 4$ and the surface $N_{a \cup b}$ obtained by cutting $N$ along $a$ and $b$ is connected (Since $N_{a}$ and $N_{b}$ are nonorientable, it is easy to see that $N_{a \cup b}$ is also nonorientable in this case.),

- or $1 \leq g \leq 3$ and the Euler characteristic of one of the connected components of $N_{a \cup b}$ is at most -2 .

We note that $N_{a \cup b}$ is disconnected if $\alpha$ is dual to $\beta$ and $1 \leq g \leq 3$. Notice also that the graph $\widetilde{X}(N)$ is defined if $g+n \geq 5$.

If $P$ and $Q$ are two points on an oriented circle $a$ on $N$, we denote by $[P, Q]_{a}$ the subarc of $a$ from $P$ to $Q$. We denote by $\overline{[P, Q]}_{a}$ the arc $[P, Q]_{a}$ with the reversed orientation. If $\alpha$ and $\beta$ are two oriented arcs such that the terminal point of $\alpha$ is the initial point of $\beta$, then we denote by $\alpha \star \beta$ the arc obtained by first traveling along $\alpha$ and then along $\beta$.

Let $a$ be an oriented circle and $b$ be any circle on $N$ intersecting $a$ at least twice. Let $P$ and $Q$ be two distinct points in $a \cap b$. We say that $b$ intersects $a$ at $P$ and $Q$ 
from the same (resp. opposite) side relative to the subarc $[P, Q]_{a}$ if two orientations of a regular neighborhood of $[P, Q]_{a}$ given by $a, b$ at the points $P$ and $Q$ agree (resp. do not agree) for any orientation of $b$. Note that this definition does not depend on the orientation of $b$.

Lemma 3.1. Let $N$ be a nonorientable surface of genus $g \geq 1$. Let a and $b$ be two one-sided oriented essential circles on $N$ such that $|a \cap b| \geq 2$. Suppose that there are two points $P$ and $Q$ in $a \cap b$ adjacent along a such that the interior of $[P, Q]_{a}$ $i$ disjoint from $b$ and that $b$ intersects $a$ at $P$ and $Q$ from the same side relative to $[P, Q]_{a}$. (There are always such points if $|a \cap b|$ is even). Then there is a circle $c$ on $N$ such that

(1) $c$ is one-sided and essential,

(2) $|c \cap b|=1$, and

(3) $|c \cap a| \leq|a \cap b|-2$.

Proof. A slight perturbation of the loop

$$
[P, Q]_{a} \star[Q, P]_{b} \star[P, Q]_{a} \star \overline{[P, Q}_{b}
$$

is the required circle $c$ (cf. Figure 4). Note that $\mathbb{Z}_{2}$-valued homology class of $c$ is equal to that of $b$, so that $c$ is one-sided and essential.
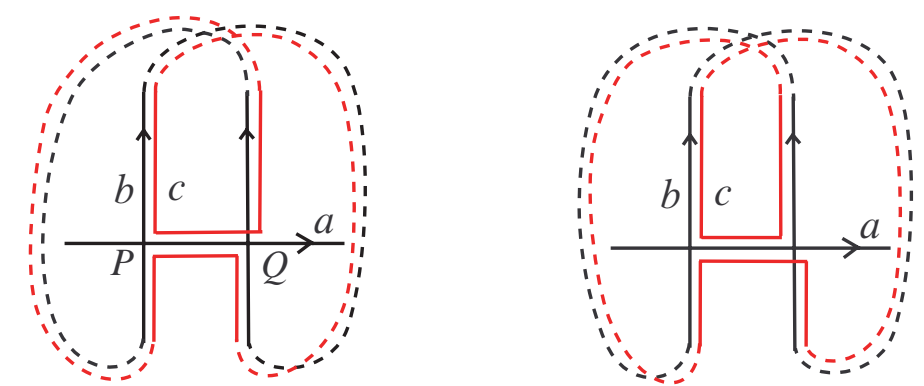

Figure 4. The construction of the circle $c$ in the case $b$ intersecting $a$ at the adjacent points $P$ and $Q$ from the same side.

Lemma 3.2. Let $N$ be a nonorientable surface of genus $g \geq 1$. Let a and $b$ be two one-sided oriented essential circles on $N$ such that $|a \cap b| \geq 3$. Suppose that there are three consecutive points $P, Q$ and $R$ in $a \cap b$ along a such that $b$ intersects $a$ from opposite sides relative to $[P, Q]_{a}$ and $[Q, R]_{a}$. Then there is one-sided circle $c$ on $N$ such that

(1) $c$ is one-sided and essential, 
(2) $|c \cap b|=3$, and

(3) $|c \cap a| \leq|a \cap b|-2$.

Proof. Note that by assumption the interiors of the arcs $[P, Q]_{a}$ and $[Q, R]_{a}$ are disjoint from $b$. By reversing the orientation of $b$ if necessary, we may assume that if we start to walk from $P$ along $b$ we reach $Q$ before $R$.

A slight perturbation of the loop

$$
[P, Q]_{b} \star[Q, R]_{a} \star[R, P]_{b} \star[P, Q]_{a} \star[Q, R]_{b} \star \overline{[P, R}_{a}
$$

is the required circle $c$. The circle $c$ is as in one of the pictures in Figure 5. Note that $\mathbb{Z}_{2}$-valued homology class of $c$ is equal to that of $b$, so that $c$ is one-sided and essential.
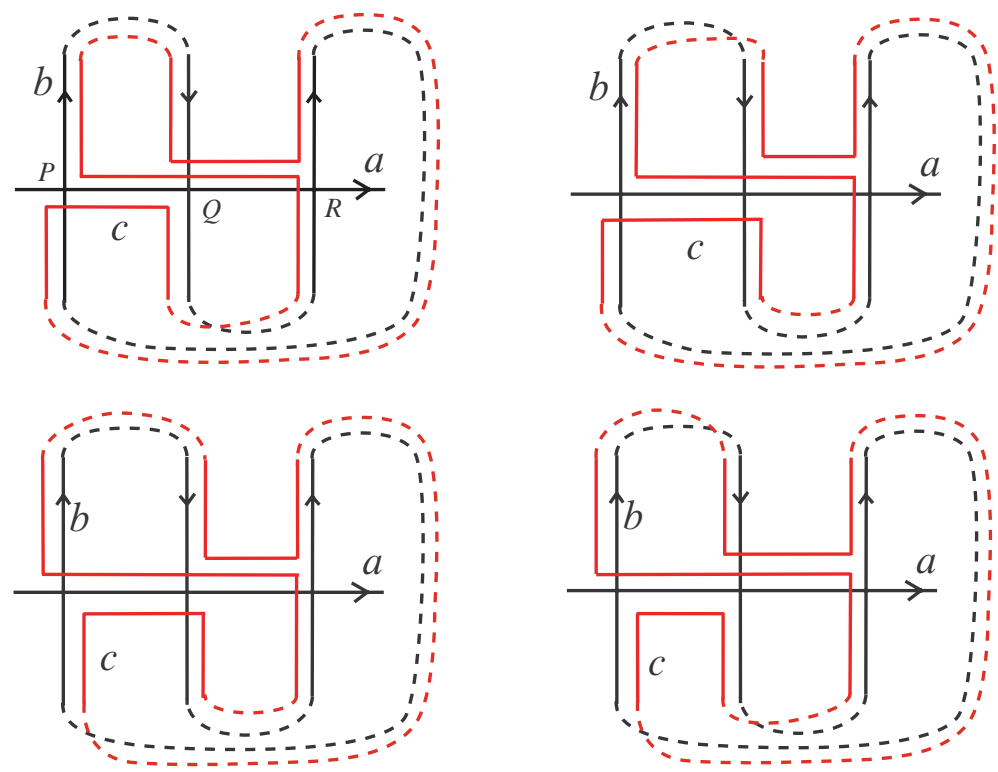

Figure 5. The construction of the circle $c$ when $b$ intersects $a$ from opposite sides at $P, Q$ and at $Q, R$.

Lemma 3.3. Let $N$ be a nonorientable surface of genus $g \geq 1$. Let $a$ and $b$ be two one-sided oriented essential circles on $N$ such that $|a \cap b|=3$. Then there is a circle $c$ on $N$ such that

(1) $c$ is one-sided and essential,

(2) $|c \cap b|=1$, and

(3) $|c \cap a| \leq 1$. 
Proof. Suppose first that $b$ intersects $a$ from the same side at two consecutive points $P$ and $Q$ on $a$. A perturbation of the loop

$$
[P, Q]_{a} \star[Q, P]_{b} \star[P, Q]_{a} \star \overline{[P, Q}_{b}
$$

is the required circle $c$ (cf. Figure 4).

Suppose now that $P, Q$ and $R$ the intersection points of $a$ and $b$ that are consecutive along $a$, and that at every two adjacent intersection points on $a, b$ intersects $a$ from the opposite side. By reversing the orientation of $b$ if necessary, we may assume that when we start to walk from $P$ along $b$ we reach $Q$ before $R$.

Let us form the following circles on the surface $N$ :

$$
\begin{aligned}
c_{1} & =[P, Q]_{b} \star \overline{[P, Q}_{a}, \\
c_{2} & =[Q, R]_{b} \star \overline{[Q, R}_{a}, \\
c_{3} & =[P, Q]_{a} \star[Q, P]_{b}, \\
c_{4} & =[P, R]_{a} \star[R, P]_{b}, \\
c_{5} & =[Q, R]_{a} \star[R, Q]_{b} .
\end{aligned}
$$

We perturb each $c_{i}$ so that they intersect $a$ and $b$ minimally. Such perturbations are shown in Figure 6.
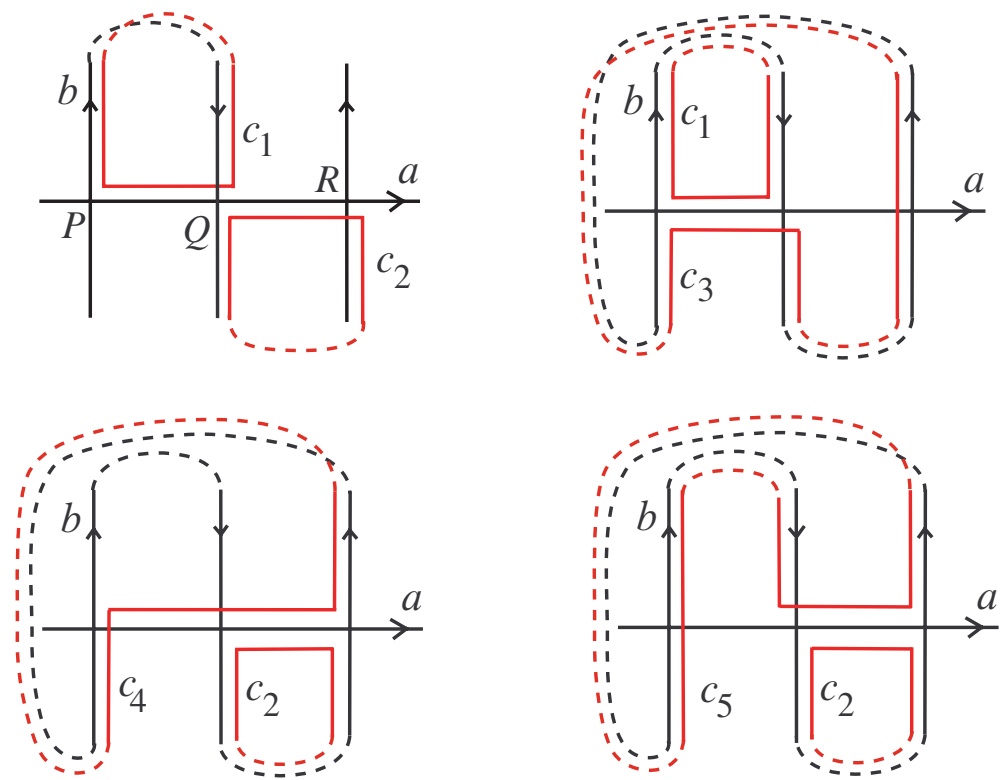

Figure 6. The circles $c_{1}, \ldots, c_{5}$.

If either $c_{1}$ or $c_{2}$ is one-sided, then it is the required circle. Note that $c_{1}$ and $c_{2}$ are both disjoint from $a$, so that they are essential whenever they are one-sided. 
Suppose that $c_{1}$ and $c_{2}$ are both two-sided. Since $\left[c_{1}\right]+\left[c_{3}\right],\left[c_{1}\right]+\left[c_{2}\right]+\left[c_{4}\right]$ and $\left[c_{2}\right]+\left[c_{5}\right]$ are equal to $[b]$ as $\mathbb{Z}_{2}$-valued homology classes, the circles $c_{3}, c_{4}$ and $c_{5}$ are all one-sided. (All homology classes in this proof are $\mathbb{Z}_{2}$-valued homology classes.) All three circles $c_{3}, c_{4}$ and $c_{5}$ satisfy (2) and (3).

If $c_{3}$ is essential, then it is the circle we are looking for. If it is not essential, then it is characteristic; that is, the complement of $c_{3}$ in $N$ is orientable. In this case $\left[c_{1}\right] \neq 0$. Then we look at the homology class of $c_{2}$.

If $\left[c_{2}\right] \neq 0$ then $c_{4}$ is essential, and if $\left[c_{2}\right]=0$ then $c_{5}$ is essential.

Corollary 3.4. Let $N$ be a nonorientable surface of genus $g \geq 1$. Let $a$ and $b$ be two one-sided essential circles on $N$ such that $|a \cap b| \geq 2$. Then there is a circle $c$ on $N$ such that

(1) $c$ is one-sided and essential, and

(2) $|c \cap b|<|a \cap b|$ and $|c \cap a|<|a \cap b|$.

Proof. Orient $a$ and $b$ arbitrarily. Then either there are two consecutive points on $a$ where $b$ intersects $a$ from the same side with respect to some subarc of $a$ (there exist such points if $|a \cap b|$ is even), or there are three consecutive points $P, Q, R$ on $a$ such that $b$ intersects $a$ from the opposite sides at $P$ and $Q$ and at $Q$ and $R$. Now the corollary follows from Lemmas 3.1, 3.2 and 3.3.

Proposition 3.5. Let $N$ be a nonorientable surface of genus 1 . The graph $X(N)$ is connected.

Proof. If $n \leq 1$ then there is only one one-sided circle on $N$, so that $X(N)$ consists of only one vertex. If $n=2$ then there are two one-sided circles on $N$ and they intersect at one point, so that $X(N)$ consists of two vertices and an edge joining them.

We assume that $n \geq 3$. Let $\alpha$ and $\beta$ be two distinct vertices of $X(N)$ represented by the circles $a$ and $b$ that intersect minimally. It suffices to prove that there is a sequence $a=c_{0}, c_{1}, c_{2}, \ldots, c_{k}=b$ of one-sided circles such that $\left|c_{i-1} \cap c_{i}\right|=1$ for all $1 \leq i \leq k$. We prove this by induction on $|a \cap b|$. Note that $|a \cap b| \geq 1$.

If $|a \cap b|=1$ then there is nothing to prove. So suppose that $|a \cap b| \geq 2$. By Corollary 3.4 there is a one-sided circle $c$ on $N$ such that $|c \cap a|<|a \cap b|$ and $|c \cap b|<|a \cap b|$. By induction, there are sequences $a=c_{0}, c_{1}, \ldots, c_{r}=c$ and $c=d_{0}, d_{1}, \ldots, d_{s}=b$ of one-sided circles with the required property. Then $a=c_{0}, c_{1}, \ldots, c_{r}=d_{0}, d_{1}, \ldots, d_{s}=b$ is the sequence we are looking for.

Proposition 3.6. Let $N$ be a nonorientable surface of genus 2 . Let $a_{1}$ and $a_{2}$ be two disjoint one-sided circles on $N$ representing the vertices $\alpha_{1}$ and $\alpha_{2}$, respectively. Then the graph $X(N)$ has two connected components such that each connected component contains exactly one of $\alpha_{1}$ and $\alpha_{2}$.

Proof. If two one-sided circles intersect at one point, then they represent the same $\mathbb{Z}_{2}$-homology class on the closed surface $\bar{N}$ of genus 2 obtained by gluing a disk 
along each hole. It follows that $\alpha_{1}$ and $\alpha_{2}$ lie on different connected components of $X(N)$ since their $\mathbb{Z}_{2}$-homology classes in $\bar{N}$ are different. We will show that every vertex in $X(N)$ is connected to either $\alpha_{1}$ or $\alpha_{2}$. This will complete the proof.

If $n=0$ then the conclusion of the proposition obviously holds since $X(N)$ consists of two points $\alpha_{1}$ and $\alpha_{2}$. So we assume that $n \geq 1$. Note that all one-sided circles on $N$ are essential.

Let $\beta$ be any vertex of $X(N)$ represented by a one-sided circle $b$ that intersects $a_{1}$ and $a_{2}$ minimally. We claim that $\beta$ is connected to either $\alpha_{1}$ or $\alpha_{2}$ in $X(N)$. We prove our claim by induction on $\left|b \cap a_{1}\right|+\left|b \cap a_{2}\right|=k$. Note that the number $k$ is odd, so that one of $\left|b \cap a_{1}\right|$ and $\left|b \cap a_{2}\right|$ is even and the other is odd.

If $k=1$ then $\left|b \cap a_{j}\right|=1$ for some $j=1,2$. Then either $\beta=\alpha_{j}$ or $\beta$ is connected to $\alpha_{j}$ by an edge. Suppose that $k \geq 3$. Then $\left|b \cap a_{j}\right|$ is even for some $j$. Without loss of generality assume that $j=1$. If $\left|b \cap a_{1}\right|=0$ then $b$ and $a_{2}$ lie on the genus one surface $N_{1}$ obtained from $N$ by cutting along $a_{1}$. By Proposition 3.5, $\beta$ can be connected to $\alpha_{2}$ (by a path all of whose vertices are represented by circles disjoint from $a_{1}$ ). If $\left|b \cap a_{1}\right| \geq 2$ then we orient $a_{1}$ and $b$ arbitrarily. There are two points $P$ and $Q$ of $\left|a_{1} \cap b\right|$ such that the interior of the subarc $[P, Q] a_{1}$ of $a_{1}$ is disjoint from $b$ and that $b$ intersects $a_{1}$ at $P$ and $Q$ from the same side relative to $[P, Q]_{a_{1}}$. Now the one-sided circle $c$ constructed in Lemma 3.1 (where $a$ is replaced by $a_{1}$ ) satisfies $|c \cap b|=1,\left|c \cap a_{1}\right| \leq\left|b \cap a_{1}\right|-2$ and $\left|c \cap a_{2}\right|=\left|b \cap a_{2}\right|$. Thus we have $\left|c \cap a_{1}\right|+\left|c \cap a_{2}\right| \leq k-2$. The vertex $\gamma$ represented by $c$ is connected to $\beta$. By induction $\gamma$ is also connected to one of $\alpha_{j}$ (in fact $\alpha_{2}$ ). This finishes the proof.

Proposition 3.7. Let $N$ be a nonorientable surface of genus 3. Let $a_{1}, a_{2}$ and $a_{3}$ be three pairwise disjoint one-sided (hence essential) circles on $N$ representing the vertices $\alpha_{1}, \alpha_{2}$ and $\alpha_{3}$, respectively. Then the graph $X(N)$ has three connected components such that each connected component contains exactly one of $\alpha_{1}, \alpha_{2}$ and $\alpha_{3}$.

Proof. As in the proof of Proposition 3.6, if two essential one-sided circles $a$ and $b$ intersect at one point, then they represent the same $\mathbb{Z}_{2}$-homology class on the closed surface $\bar{N}$ of genus 3 obtained by gluing a disk along each hole. It follows that $\alpha_{1}$, $\alpha_{2}$ and $\alpha_{3}$ lie on different connected components of $X(N)$ since their $\mathbb{Z}_{2}$-homology classes in $\bar{N}$ are different. We show that every vertex in $X(N)$ is connected to either $\alpha_{1}$, or $\alpha_{2}$, or $\alpha_{3}$, which will complete the proof.

Let $\beta$ be any vertex of $X(N)$ represented by an essential one-sided circle $b$ that intersects $a_{1}, a_{2}$ and $a_{3}$ minimally. We claim that $\beta$ is connected in $X(N)$ to one of $\alpha_{1}, \alpha_{2}$ or $\alpha_{3}$. We prove our claim by induction on $\left|b \cap a_{1}\right|+\left|b \cap a_{2}\right|+\left|b \cap a_{3}\right|=k$. Note that $k \geq 1$.

Since $b$ is essential, its $\mathbb{Z}_{2}$-homology class in $\bar{N}$ is one of $\left[a_{1}\right]$, [ $\left.a_{2}\right]$, or $\left[a_{3}\right]$. Hence, $\left|b \cap a_{j}\right|$ is odd for some $j=1,2,3$ and $\left|b \cap a_{i}\right|$ is even for $i \neq j$. In particular $k$ is odd. 
If $k=1$ then $\left|b \cap a_{j}\right|=1$ for some $j=1,2,3$. Then either $\beta=\alpha_{j}$ or $\beta$ is connected to $\alpha_{j}$ by an edge. Suppose that $k \geq 3$. Then $\left|b \cap a_{j}\right|$ is even for some $j$ (There are two such $j$ ). Without loss of generality assume that $j=1$. If $\left|b \cap a_{1}\right|=0$ then the curves $b, a_{2}$ and $a_{3}$ lie on the surface $N_{1}$ obtained by cutting $N$ along $a_{1}$. Since $N_{1}$ is of genus two, by Proposition 3.6, $\beta$ can be connected to $\alpha_{2}$ or $\alpha_{3}$ (by a path all of whose vertices are represented by circles disjoint from $a_{1}$ ). If $\left|b \cap a_{1}\right| \geq 2$ then we orient $a_{1}$ and $b$ arbitrarily. There are two points $P$ and $Q$ of $\left|a_{1} \cap b\right|$ such that the interior of the subarc $[P, Q]_{a_{1}}$ of $a_{1}$ is disjoint from $b$ and that $b$ intersects $a_{1}$ at $P$ and $Q$ from the same side relative to $[P, Q]_{a_{1}}$. Now the one-sided circle $c$ constructed in Lemma 3.1 (where $a$ is replaced by $a_{1}$ ) is essential and satisfies $|c \cap b|=1,\left|c \cap a_{1}\right| \leq\left|b \cap a_{1}\right|-2,\left|c \cap a_{2}\right|=\left|b \cap a_{2}\right|$ and $\left|c \cap a_{3}\right|=\left|b \cap a_{3}\right|$. Thus we have $\left|c \cap a_{1}\right|+\left|c \cap a_{2}\right|+\left|c \cap a_{3}\right| \leq k-2$. By induction the vertex $\gamma$ is connected to one of $\alpha_{j}$. Since $\gamma$ is also connected to $\beta$ by an edge, the proof is complete.

Proposition 3.8. Let $N$ be a nonorientable surface of genus $g \geq 4$. Then the graph $X(N)$ is connected.

Proof. Let $\alpha$ and $\beta$ be two vertices of $X(N)$ represented by $a$ and $b$ respectively that intersect minimally. We claim that there exists a sequence $a=a_{0}, a_{1}, a_{2}, \ldots, a_{m}=$ $b$ of essential one-sided circles such that the circle $a_{i-1}$ intersects $a_{i}$ transversely at one point. The proof follows from this. We prove this claim by induction on $k=|a \cap b|$.

Suppose first that $k=0$, so that $a$ and $b$ are disjoint. Clearly, the surface $N_{a \cup b}$ obtained by cutting $N$ along $a$ and $b$ is connected. If $N_{a \cup b}$ is nonorientable, there is another essential circle $d$ disjoint from both $a$ and $b$ such that the complement of $a \cup b \cup d$ is nonorientable. Then it easy to find an essential circle $c$ representing the $\mathbb{Z}_{2}$-homology class $[a]+[b]+[d]$ such that $c$ intersects each of $a$ and $b$ at only one point (cf. Figure 7 (i)). Then $a, c, b$ is the required sequence. If $N_{a \cup b}$ is orientable, let $e$ be any two-sided nonseparating circle on $N$ disjoint from $a$ and $b$. One can easily find a one-sided essential circle $d$ representing the $\mathbb{Z}_{2}$-homology class $[b]+[e]$ such that $d$ intersects $b$ at one point, $d$ is disjoint from $a$, and the surface $N_{d \cup a}$ is nonorientable (cf. Figure 7 (ii)). By the previous case, there is an essential circle $c$ such that $a, c, d, b$ is the sequence we are looking for.

If $k=1$ there is nothing to prove.

Suppose now that $k \geq 2$. By Corollary 3.4, there exists an essential one-sided circle $c$ such that $|c \cap a|<|a \cap b|$ and $|c \cap b|<|a \cap b|$. By induction, there are two sequences of essential one-sided circles $a=a_{0}, a_{1}, \ldots, a_{k}=c$ and $c=$ $c_{0}, c_{1}, \ldots, c_{l}=b$ such that any two adjacent circles in the sequences intersect once. Then $a=a_{0}, a_{1}, \ldots, a_{k}=c=c_{0}, c_{1}, \ldots, c_{l}=b$ is the required sequence.

This completes the proof of the proposition.

We collect the above results in the next theorem. 


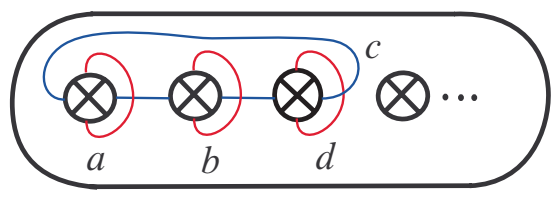

(i)

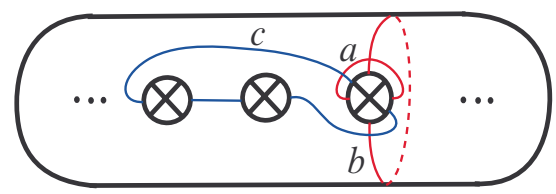

(iii)

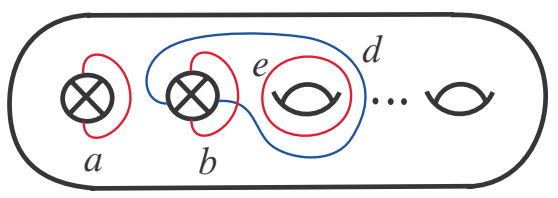

(ii)

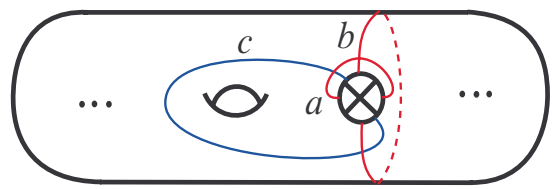

(iv)

Figure 7. The surface $N_{a \cup b}$ is nonorientable in (i) and orientable in (ii). The complements of $a \cup c$ and $b \cup c$ are connected in (iii) and in (iv).

Theorem 3.9. Let $N$ be a nonorientable surface of genus $g$. Then the graph $X(N)$

(1) is connected if $g=1$ or $g \geq 4$,

(2) has $g$ connected components if $g=2$ or $g=3$, one for each essential generator of $H_{1}\left(\bar{N} ; \mathbb{Z}_{2}\right)$, where $\bar{N}$ is the closed nonorientable surface of genus $g$ obtained from $N$ by gluing a disk along each hole.

Theorem 3.10. Let $g+n \geq 5$ and let $N$ be a nonorientable surface of genus $g$ with $n$ holes. Then the subgraph $\tilde{X}(N)$ of $X(N)$

(1) is connected if $g=1$ or $g \geq 4$,

(2) has $g$ connected components if $g=2$ or $g=3$.

Proof. Let $\alpha$ and $\beta$ be two distinct vertices of $\tilde{X}(N)$ such that $\alpha$ is connected to $\beta$ by an edge in $X(N)$. By Theorem 3.9, it suffices to prove that $\alpha$ is connected to $\beta$ in $\widetilde{X}(N)$. Choose circles $a \in \alpha$ and $b \in \beta$ with $|a \cap b|=1$. Let $N_{a \cup b}$ denote the surface obtained by cutting $N$ along $a \cup b$.

Suppose first that $g \geq 4$. If $N_{a \cup b}$ is connected, there is nothing to prove. So suppose that $N_{a \cup b}$ is disconnected. A regular neighborhood of $a \cup b$ is a nonorientable surface of genus one with two boundary components. Since $g \geq 4$, one of the components of $N_{a \cup b}$ is either nonorientable of genus at least two or orientable of genus at least one. In either case, one can find a one-sided essential circle $c$, as shown in Figure 7 (iii) and (iv), such that $c$ intersects both $a$ and $b$ at one point and the surfaces $N_{a \cup c}$ and $N_{b \cup c}$ are connected. If $\gamma$ is the isotopy class of $c$, then the vertex $\gamma$ is connected to both $\alpha$ and $\beta$ by an edge in $\widetilde{X}(N)$. 
Suppose now that $1 \leq g \leq 3$. Notice that $N_{a \cup b}$ has two connected components and its Euler characteristic is $3-g-n$, which is less than or equal to -2 . If $g+n \geq 6$ then the Euler characteristic of at least one of the components of $N_{a \cup b}$ is less than -1 , so that $\alpha$ and $\beta$ are connected by an edge in $\tilde{X}(N)$. Hence, we have $X(N)=\widetilde{X}(N)$ when $g+n \geq 6$.

If $g+n=5$ and if the Euler characteristics of both components of $N_{a \cup b}$ are -1 then there is a one-sided essential circle $c$ intersecting both $a$ and $b$ at one point such that one of the components has Euler characteristic -2 (cf. Figure 8). If $\gamma$ denotes the isotopy class of $c$, then $\gamma$ is connected to both $\alpha$ and $\beta$ by an edge in $\tilde{X}(N)$.

This finishes the proof of the theorem.

$b$

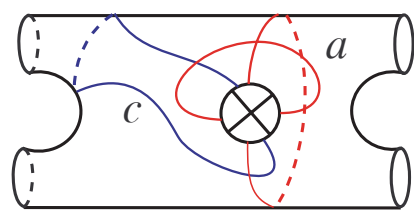

$b$

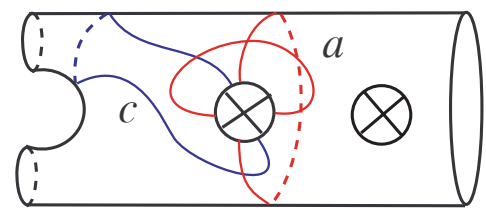

$b$

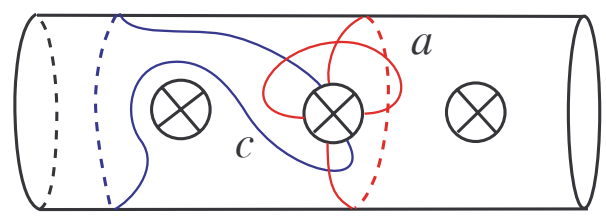

$b$

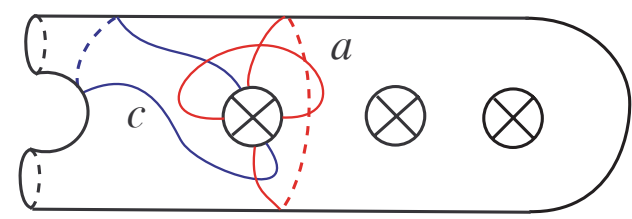

Figure 8. The isotopy class of $c$ is connected to the isotopy classes of $a$ and $b$ by an edge in $\widetilde{X}(N)$.

\section{Classification of curve complexes}

We prove in this section that two surfaces (orientable or nonorientable) are diffeomorphic if and only if their curve complexes are isomorphic provided that these surfaces do not form one of the following pairs: A sphere with four holes and a torus with at most one hole, a sphere with five holes and a torus with two holes, or a sphere with six holes and a closed orientable surface of genus two. Here, we assume that the curve complexes are nonempty.

Throughout this section, $N$ will denote a compact connected nonorientable surface of genus $g$ with $n$ holes. 
Remark 4.1. If $S$ is a sphere with four holes or a torus with at most one hole, then the curve complex $C(S)$ is countably infinite discrete. Therefore, the curve complexes of these surfaces are isomorphic. Similarly the curve complex of a sphere with five (resp. six) holes and that of a torus with two holes (resp. a closed orientable surface of genus two) are isomorphic.

Let us call a pair $\left(\Sigma_{1}, \Sigma_{2}\right)$ of surfaces an exceptional pair if $\Sigma_{1}$ and $\Sigma_{2}$ are the surfaces above such that they are not diffeomorphic but their curve complexes are isomorphic.

Theorem 4.2 ([15], [13]). Let $S$ and $\Sigma$ be two connected orientable surfaces such that $(S, \Sigma)$ is not exceptional pair. The curve complexes $C(S)$ and $C(\Sigma)$ are isomorphic if and only if $S$ and $\Sigma$ are diffeomorphic.

Theorem 4.2 was proved in [13] for surfaces of genus at most one and in [15] in the above generality. The proof of this theorem follows from Theorem 3, since the extended mapping class groups of nondiffeomorphic surfaces are not isomorphic.

If $N$ and $S$ are two surfaces and if $\varphi: C(N) \rightarrow C(S)$ is an isomorphism, then $\varphi$ induces an isomorphism $L(\alpha) \rightarrow L(\varphi(\alpha))$ for any vertex $\alpha$ in $C(N)$. We will use this fact.

Lemma 4.3. Let $N$ be a connected nonorientable surface and $S$ a connected orientable surface. Then $C(N)$ and $C(S)$ are not isomorphic.

Proof. If the genus of $N$ is at least two, then there are maximal simplices of different dimensions in $C(N)$. On the other hand, all maximal simplices in $C(S)$ have the same dimension. Hence, $C(N)$ and $C(S)$ cannot be isomorphic.

Suppose that $N$ is of genus one with $n$ holes. If $n \in\{0,1,2\}$ then $C(N)$ is nonempty finite, but $C(S)$ is not.

If $n \geq 3$, then let $a$ be a two-sided circle on $N$ such that the components of $N_{a}$ are a sphere $D$ with $n$ holes and a nonorientable surface $M$ of genus 1 with two holes. Let $\alpha$ denote the isotopy class of $a$. Then $L(\alpha)$ is isomorphic to the join $C(M) \star C(D)$, where $C(M)$ consists of only two points. The link of no vertex in the curve complex of an orientable surface has such a decomposition. Therefore, $C(N)$ cannot be isomorphic to $C(S)$.

Lemma 4.4. Let $r \geq 1$. If $N$ is a connected nonorientable surface of genus $g=2 r$ with $n$ holes and $M$ is a connected nonorientable surface of genus $2 r+1$, then $C(N)$ and $C(M)$ are not isomorphic.

Proof. Note that the curve complex of a connected surface is isomorphic to the disjoint union of a closed interval (resp. infinite line) and a point if and only if the surface is a Klein bottle (resp. a Klein bottle with one hole). It follows that if $N$ is a Klein bottle with at most one hole, then $C(N)$ is not isomorphic to $C(M)$. Hence, we may assume that $N$ is not a Klein bottle with at most one hole. 
Suppose that there is an isomorphism $\varphi: C(N) \rightarrow C(M)$. We will arrive at a contradiction.

Let us say that a vertex $\alpha$ on a nonorientable surface $\Sigma$ bounds a Klein bottle if $\alpha$ is represented by a two-sided separating circle $a$ such that one of the components of $\Sigma_{a}$, the surface obtained by cutting $N$ along $a$, is a Klein bottle $K$ with one hole. Observe that if $\alpha$ bounds a Klein bottle $K$, then the link $L(\alpha)$ of $\alpha$ is isomorphic to the join $C(K) \star C\left(K^{\prime}\right)$ for some surface $K^{\prime}$. Conversely, if the link $L(\alpha)$ is isomorphic to $C(K) \star C\left(K^{\prime}\right)$, then $\alpha$ bounds a Klein bottle.

It follows from this observation that if $\alpha$ bounds a Klein bottle on $N$, then $\varphi(\alpha)$ bounds a Klein bottle on $M$.

Suppose that $(g, n) \neq(4,0)$. Let $\alpha_{1}, \alpha_{2}, \ldots, \alpha_{r}$ be vertices in $C(N)$ such that each $\alpha_{i}$ bounds a Klein bottle and $\Delta=\left\{\alpha_{1}, \alpha_{2}, \ldots, \alpha_{r}\right\}$ is a simplex of dimension $r-1$. Then $\varphi(\Delta)$ is a simplex of dimension $r-1$ in $C(M)$. The link of $\Delta$ is equal to $L\left(\alpha_{1}\right) \cap L\left(\alpha_{2}\right) \cap \cdots \cap L\left(\alpha_{r}\right)$, which is isomorphic to

$$
C\left(K_{1}\right) \star C\left(K_{2}\right) \star \cdots \star C\left(K_{r}\right) \star C\left(S_{n+r}\right),
$$

where each $K_{i}$ is a Klein bottle with one hole and $S_{n+r}$ is a sphere with $n+r$ holes. On the other hand, the link of $\varphi(\Delta)$ is isomorphic to

$$
C\left(K_{1}\right) \star C\left(K_{2}\right) \star \cdots \star C\left(K_{r}\right) \star C(P),
$$

for some nonorientable surface $P$ of genus one. It is easy now to conclude that $C\left(S_{n+r}\right)$ is isomorphic to $C(P)$, which is impossible by Lemma 4.3.

Suppose now that $(g, n)=(4,0)$. Let $a$ be a circle bounding a Klein bottle and let $\alpha$ be the isotopy class of $a$. The link of $\alpha$ is equal to

$$
C\left(K_{1}\right) \star C\left(K_{2}\right)
$$

where each $K_{i}$ is a Klein bottle with one hole. Hence, the link of $\varphi(\alpha)$ is isomorphic to

$$
C\left(K_{1}\right) \star C\left(K_{2}\right)
$$

But there is no such a vertex in $C(M)$.

This contradiction finishes the proof of the lemma.

Lemma 4.5. Let $N$ (resp. $M$ ) be a connected nonorientable surface of genus $g$ (resp. $h$ ) with $n$ (resp. $m$ ) holes. If $C(N)$ and $C(M)$ are isomorphic, then $g=h$ and $n=m$.

Proof. Suppose that $C(N)$ and $C(M)$ are isomorphic. Without loss of generality, we assume that $g \leq h$.

By Proposition 2.3, we get $\left\lceil\frac{g}{2}\right\rceil=\left\lceil\frac{h}{2}\right\rceil$. Recall that $\left\lceil\frac{g}{2}\right\rceil$ denotes the smallest integer bigger than $\frac{g}{2}$. It follows that either $g=h$, or $g$ is even and $h=g+1$. By Lemma 4.4, the latter case is not possible. Hence, $g=h$. By looking at the dimensions of the complexes one can deduce that $n=m$. 
We collect the results of this section in the next theorem.

Theorem 4.6. Let $\Sigma_{1}$ and $\Sigma_{2}$ be two connected surfaces such that $C\left(\Sigma_{1}\right)$ and $C\left(\Sigma_{2}\right)$ are nonempty and $\left(\Sigma_{1}, \Sigma_{2}\right)$ is not an exceptional pair. Then the curve complexes $C\left(\Sigma_{1}\right)$ and $C\left(\Sigma_{2}\right)$ are isomorphic if and only if $\Sigma_{1}$ and $\Sigma_{2}$ are diffeomorphic.

\section{Topological types of vertices in $C(N)$}

We prove in this section that automorphisms of the curve complex preserve the topological types of vertices. Recall that two vertices in $C(N)$ have the same topological type if there is a diffeomorphism mapping one to the other.

Theorem 5.1. Let $N$ be a connected nonorientable surface of genus $g$ with $n$ holes. The group Aut $C(N)$ preserves the topological types of the vertices of $C(N)$. That is, if $\varphi$ is an automorphism of $C(N)$ and if $\alpha$ is a vertex of $C(N)$, then $\alpha$ and $\varphi(\alpha)$ have the same topological type.

Proof. Let us denote by $N_{g}^{k}$ (resp. $S_{g}^{k}$ ) the nonorientable (resp. orientable) surface of genus $g$ with $k$ holes.

Let $\alpha$ be a vertex in $C(N)$ represented by a circle $a$. The link $L(\alpha)$ of $\alpha$ is isomorphic to the following complex:

- If $\alpha$ is one-sided and essential ( $N_{a}$ is nonorientable), then the link $L(\alpha)$ is isomorphic to the curve complex $C\left(N_{g-1}^{n+1}\right)$.

- If $\alpha$ is one-sided and if $N_{a}$ is orientable (this occurs when $g$ is odd), then the link $L(\alpha)$ is isomorphic to $C\left(S_{(g-1) / 2}^{n+1}\right)$.

- If $\alpha$ is two-sided nonseparating and if $N_{a}$ is nonorientable, then $L(\alpha)$ is isomorphic to $C\left(N_{g-2}^{n+2}\right)$.

- If $\alpha$ is two-sided nonseparating and if $N_{a}$ is orientable (this occurs if $g$ is even), then the link $L(\alpha)$ is isomorphic to $C\left(S_{(g-2) / 2}^{n+2}\right)$.

- If $\alpha$ bounds a disk with two holes, then the link $L(\alpha)$ is isomorphic to $C\left(N_{g}^{n-1}\right)$.

- If $\alpha$ is separating and if the connected components of $N_{a}$ are $N_{k}^{l+1}$ and $N_{g-k}^{n-l+1}$, then the link $L(\alpha)$ is isomorphic to the join $C\left(N_{k}^{l+1}\right) \star C\left(N_{g-k}^{n-l+1}\right)$.

- If $\alpha$ is separating and if the connected components of $N_{a}$ are $S_{k}^{l+1}$ and $N_{g-2 k}^{n-l+1}$, then the link $L(\alpha)$ is isomorphic to $C\left(S_{k}^{l+1}\right) \star C\left(N_{g-2 k}^{n-l+1}\right)$.

Since the links of vertices of different topological types are pairwise nonisomorphic, the proof of the theorem follows. 


\section{Injectivity of $\operatorname{Mod}(N) \rightarrow \operatorname{Aut} C(N)$}

We show in this section that the natural $\operatorname{map} \operatorname{Mod}(N) \rightarrow \operatorname{Aut} C(N)$ is injective. As in the orientable case, the injectivity of this map is the easier part of the proof. The proof will basically follow from the corresponding result in orientable case, Theorem 3, by an induction on the genus of the surface. For this purpose we consider the sphere as a nonorientable surface of genus zero.

Theorem 6.1. Let $N$ be a connected nonorientable surface of genus $g \geq 0$ with $n$ holes. Suppose that $g+n \geq 5$. The natural map $\operatorname{Mod}(N) \rightarrow$ Aut $C(N)$ is injective.

Proof. The proof is by induction on the genus $g$. Theorem 6.1 holds true in the case $g=0$, by Theorem 3. Suppose now that $g \geq 1$, and that $f$ is an element of $\operatorname{Mod}(N)$ acting trivially on $C(N)$, so that it fixes every vertex of $C(N)$. Let $F$ be a diffeomorphism representing $f$.

Let $a$ be a one-sided essential circle on $N$, so that the surface $N_{a}$ obtained by cutting $N$ along $a$ is a nonorientable surface of genus $g-1$ with $n+1$ boundary components. Denote by $\alpha$ the isotopy class of $a$. Since $f(\alpha)=\alpha, F(a)$ is isotopic to $a$. Choose a diffeomorphism $G$ isotopic to identity such that $G(F(a))=a$. Let $H=G \circ F$. Let $H_{a}$ be the diffeomorphism $N_{a} \rightarrow N_{a}$ induced by $H$. Clearly, the isotopy class of $H_{a}$ acts trivially on $C\left(N_{a}\right)$. Note that the genus and the number of boundary components of $N_{a}$ satisfy $(g-1)+(n+1) \geq 5$.

By induction, the diffeomorphism $H_{a}$ is isotopic to the identity of $N_{a}$. One can choose the isotopy so that it induces an isotopy $N \rightarrow N$ between $H$ and the identity. Hence, the diffeomorphism $F$, which is isotopic to $H$, is isotopic to the identity, so that the $\operatorname{map} \operatorname{Mod}(N) \rightarrow$ Aut $C(N)$ is injective.

\section{Surjectivity of $\operatorname{Mod}(N) \rightarrow \operatorname{Aut} C(N)$}

In this section, it is again convenient to consider the sphere as a nonorientable surface of genus zero. In order to prove that the natural map $\operatorname{Mod}(N) \rightarrow \operatorname{Aut} C(N)$ is surjective, we induct the genus of the surface. The initial case $g=0$ is already known by Theorem 3 .

Before completing the proof, we need some preparation.

\subsection{Preparation for the surjectivity}

Lemma 7.1. Let $g+n \geq 4$ and let $N$ be a compact connected nonorientable surface of genus $g \geq 0$ with $n$ holes. Let $\partial$ be one of the boundary components of $N$ and let $F: N \rightarrow N$ be a diffeomorphism with the property that $F(\partial)=\partial$. If $F(c)$ is isotopic to $c$ for all nontrivial circles $c$ on $N$, then $F$ is isotopic to the identity. If $F$ and the isotopies are assumed to be the identity on $\partial$, then $F$ is isotopic to a power of the Dehn twist $t_{\partial}$. 
Proof. We prove our lemma by induction on the genus $g$. Once we prove the first assertion, the second is clear.

As the first step of the induction, suppose that $g=0$, so that $N$ is a sphere with $n \geq 4$ holes.

Let $n \geq 5$, so that $N$ is a sphere with $n$ holes. By assumption, the isotopy class of $F$ acts trivially on $C(N)$. By Theorem 3, $F$ is isotopic to the identity.

Let $n=4$, so that $N$ is a sphere with four holes. Let $\delta$ be a boundary component of $N$ different from $\partial$. Choose a nontrivial circle $c$ such that $\partial$ and $\delta$ lie on the same connected component of $N_{c}$, which is a pair of pants. Since $F(c)$ is isotopic to $c$ and $F(\partial)=\partial$, it follows that $F(\delta)=\delta$. It follows that $F$ preserves all boundary components of $N$. Since it fixes the isotopy class of each circle, it follows that $F$ is isotopic to the identity.

Suppose now that $N$ is a nonorientable surface of genus $g \geq 1$ with $n$ holes. Let $a$ be a one-sided circle on $N$. Since $F(a)$ is isotopic to $a$ by composing $F$ with a diffeomorphism isotopic to the identity we may assume that $F(a)=a$. Then $F$ induces a diffeomorphism $H: N_{a} \rightarrow N_{a}$ such that $H(\partial)=\partial$. By induction hypothesis, $H$ is isotopic to the identity on $N_{a}$.

Let $\delta$ denote the boundary component of $N_{a}$ so that $N$ is obtained from $N_{a}$ by identifying the antipodal points on $\delta$. Orient $\delta$ arbitrarily. Since $H$ is isotopic to the identity, the diffeomorphism $H$ preserves the orientation of $\delta$. The isotopy between $H$ and the identity can be chosen in such a way that it induces an isotopy between $F$ and the identity of $N$.

Lemma 7.2. Let $N$ be a sphere with three holes and let $\partial$ be one of the boundary components of $N$ oriented arbitrarily. Let $F: N \rightarrow N$ be a diffeomorphism with the property that $F(\partial)=\partial$ and $F$ preserves the orientation of $\partial$. Then either $F$ is isotopic to the identity or to the half twist $\sigma$ interchanging the other two boundary components. If $F$ and the isotopies are assumed to be the identity on $\partial$, then $F$ is isotopic to a power of $\sigma$.

Proof. The lemma follows easily from the description of the mapping class group of $N$, which is well known.

Lemma 7.3. Let $N$ be a nonorientable surface of genus one with two holes or a Klein bottle with one hole. Let $\partial$ be one of the boundary components of $N$ oriented arbitrarily and let $F: N \rightarrow N$ be a diffeomorphism with the property that $F(\partial)=\partial$ and $F$ preserves the orientation of $\partial$. If $F(c)$ is isotopic to $c$ for all nontrivial circles $c$ on $N$, then $F$ is isotopic to the identity. If $F$ and the isotopies are assumed to be the identity on $\partial$, then $F$ is isotopic to a power of the Dehn twist $t_{\partial}$.

Proof. The second conclusion follows from the first. Suppose first that $N$ is a projective plane with two holes. The mapping class group of $N$ preserving each hole consists of four elements, $v_{1}, v_{2}, v_{1} v_{2}$ and the identity (cf. Theorem 2.4). The mapping classes $v_{1}$ and $v_{2}$ do not act trivially on the curve complex and $v_{1} v_{2}$ reverses 
the orientations of both boundary components of $N$. Hence, $F$ must be isotopic to the identity.

Suppose now that $N$ is a Klein bottle with one hole. Let $a_{1}$ and $a_{2}$ be two distinct one-sided disjoint circles on $N$. Since $F\left(a_{i}\right)$ is isotopic to $a_{i}$, by composing $F$ with a diffeomorphism isotopic to the identity, we may assume that $F\left(a_{i}\right)=a_{i}$. Cutting $N$ along the one-sided circles $a_{1}$ and $a_{2}$, we get a pair of pants $P$ such that $\bar{F}$ preserves the orientation of $\partial$, where $\bar{F}$ is the diffeomorphism of $P$ induced from $F$. Hence, $\bar{F}$ is orientation-preserving.

Let $\delta_{1}$ and $\delta_{2}$ be the boundary components of $P$ coming from $a_{1}$ and $a_{2}$ respectively. Orient $\delta_{1}$ and $\delta_{2}$ arbitrarily. Since $\bar{F}$ is orientation-preserving, it preserves the orientation of each $\delta_{i}$ and is isotopic to the identity. We can choose the isotopy in such a way that it respects the antipodal points on each $\delta_{i}$, so that it gives an isotopy between $F$ and the identity on $N$.

7.2. The main step in the proof. The following theorem is a crucial step in the proof of the surjectivity of the natural map $\operatorname{Mod}(N) \rightarrow$ Aut $C(N)$.

Theorem 7.4. Let $g+n \geq 5$ and let $N$ be a compact connected nonorientable surface of genus $g \geq 1$ with $n$ holes. Let $\alpha$ and $\beta$ be two distinct one-sided essential vertices in $C(N)$ such that they are connected by an edge in $\tilde{X}(N)$. Let h be a mapping class such that $h(\gamma)=\gamma$ for all vertices $\gamma$ in $(\operatorname{St}(\alpha) \cup D(\alpha)) \cap(\operatorname{St}(\beta) \cup D(\beta))$. Then $h$ is equal to the identity.

Proof. We refer to Section 3 for the definition of $\widetilde{X}(N)$. Recall that for a one-sided vertex $\gamma$, the set $D(\gamma)$ consists of the vertices of $C(N)$ dual to $\gamma$.

Let $a$ and $b$ be two circles representing $\alpha$ and $\beta$, respectively, such that $a$ intersects $b$ transversely at one point. Let $N_{0}$ be a regular neighborhood of $a \cup b$. Note that $N_{0}$ is a real projective plane with two boundary components, say $d_{1}$ and $d_{2}$. Let us orient $d_{1}$ and $d_{2}$ arbitrarily.

If $g \geq 4$ then the surface obtained by cutting $N$ along $d_{1} \cup d_{2}$ is a disjoint union of $N_{0}$ and a nonorientable surface $N_{1}$. If $1 \leq g \leq 3$, then the surface obtained by cutting $N$ along $d_{1} \cup d_{2}$ is a disjoint union of $N_{0}$ and two other surfaces $N_{1}$ and $N_{2}$. Suppose that, for $i=1,2$, the surface $N_{i}$ is of genus $g_{i}$ with $n_{i}$ holes. Without loss of generality, we may assume that $g_{1}+n_{1} \geq g_{2}+n_{2}$. Note that $N_{i}$ cannot be an orientable surface of positive genus.

Let $\partial_{i}$ be the isotopy class of $d_{i}$ whenever $d_{i}$ is nontrivial. The vertex $\partial_{i}$ is contained in $(\operatorname{St}(\alpha) \cup D(\alpha)) \cap(\operatorname{St}(\beta) \cup D(\beta))$, so that $h\left(\partial_{i}\right)=\partial_{i}$. Let $H$ be a diffeomorphism in the class $h$ such that $H\left(d_{i}\right)=d_{i}$. Then $H$ induces diffeomorphisms $H_{j}: N_{j} \rightarrow N_{j}$ for each $j=0,1,2$. We denote the class of $H_{j}$ by $h_{j}$.

Suppose first that $g \geq 4$. By assumption, $N_{1}$ is connected and nonorientable. The surface $N_{1}$ has two distinguished boundary components $d_{1}$ and $d_{2}$ and $H_{1}\left(d_{i}\right)=d_{i}$. It is easy to see that the genus of $N_{1}$ is $g-3 \geq 1$. Since $g+n \geq 5$, we have $g_{1}+n_{1}=$ $(g-3)+(n+2) \geq 4$. By Lemma 7.1, $H_{1}$ is isotopic to the identity. In particular, $H$ 
preserves the orientation of each $d_{i}$. By Lemma 7.3, $H_{0}$ is also isotopic to the identity. It follows that $H$ is isotopic to a product $\left(t_{d_{1}}\right)^{m_{1}}\left(t_{d_{2}}\right)^{m_{2}}$ for some integers $m_{1}$ and $m_{2}$, where $t_{d_{i}}$ denotes a Dehn twist about $d_{i}$. It is easy to find a one-sided essential circle $c$ on $N$ which intersects $d_{1}, d_{2}$ and $a$ only once and is disjoint from $b$ (cf. Figure 9). The isotopy class $\gamma$ of $c$ is contained in $(\operatorname{St}(\alpha) \cup D(\alpha)) \cap(\operatorname{St}(\beta) \cup D(\beta))$. Since $h(\gamma)=\gamma$, we must have $m_{1}=m_{2}=0$. Hence, $h$ is the identity.

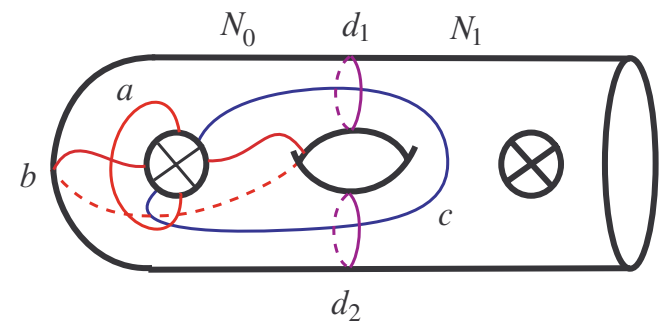

Figure 9. The circle $c$ is the case $g \geq 4$.

Suppose now that $1 \leq g \leq 3$. We may assume that $d_{i}$ is a boundary component of $N_{i}$. If one of $N_{i}$, say $N_{2}$, is an annulus, then we glue it to $N_{0}$, so that we assume that the complement of $d_{1} \cup d_{2}$ has two connected components, $N_{0}$ and $N_{1}$. Since $g_{1}+n_{1} \geq g_{2}+n_{2}$, the Euler characteristic of $N_{1}$ is less than or equal to the Euler characteristic of $N_{2}$. Since $\alpha$ and $\beta$ are connected by an edge in $\widetilde{X}(N)$, the Euler characteristic of $N_{1}$ is at most -2 , so that $g_{1}+n_{1} \geq 4$.

By Lemma 7.1, $H_{1}$ is isotopic to the identity. In particular, $H$ preserves the orientation of $d_{1}$. By Lemma 7.3, $H_{0}$ is isotopic to the identity, and hence $H$ preserves the orientation of $d_{2}$ as well. By composing $H$ with a diffeomorphism isotopic to the identity we may assume that $H$ is the identity on $N_{0}$, so that $H_{1}$ is isotopic to a power of the Dehn twist $t_{d_{1}}$ about $d_{1}$.

If $g_{2}+n_{2} \geq 3$ and $\left(g_{2}, n_{2}\right) \neq(0,3)$ then $H_{2}$ is isotopic to a power of the Dehn twist $t_{d_{2}}$ by Lemmas 7.1 and 7.3. Hence, $H$ is isotopic to $\left(t_{d_{1}}\right)^{m_{1}}\left(t_{d_{2}}\right)^{m_{2}}$.

If $\left(g_{2}, n_{2}\right)=(0,3)$ then $H_{2}$ is isotopic to a power of the half twist $\sigma$ by Lemma 7.2, where $\sigma$ interchanges two boundary components of $N_{2}$ other than $d_{2}$. Hence, $H$ is isotopic to $\left(t_{d_{1}}\right)^{m_{1}} \sigma^{m_{2}}$.

If $\left(g_{2}, n_{2}\right)=(1,1)$ then $H_{2}$ is isotopic to the identity since the mapping class group of $N_{2}$ is trivial. Hence, $H$ is isotopic to $\left(t_{d_{1}}\right)^{m_{1}}$.

It is easy to find a one-sided circle $c_{1}$ intersecting $d_{1}$ such that the isotopy class $\gamma_{1}$ of $c_{1}$ is contained in $(\operatorname{St}(\alpha) \cup D(\alpha)) \cap(\operatorname{St}(\beta) \cup D(\beta))$ (cf. Figure 10). Since $H\left(c_{1}\right)$ is isotopic to the identity by hypothesis, we conclude that $m_{1}=0$. Similarly, one may conclude that $m_{2}=0$ by using the circle $c_{2}$ on $N$ intersecting $\partial_{2}$ and that its isotopy class is contained in $(\operatorname{St}(\alpha) \cup D(\alpha)) \cap(\operatorname{St}(\beta) \cup D(\beta))$. 

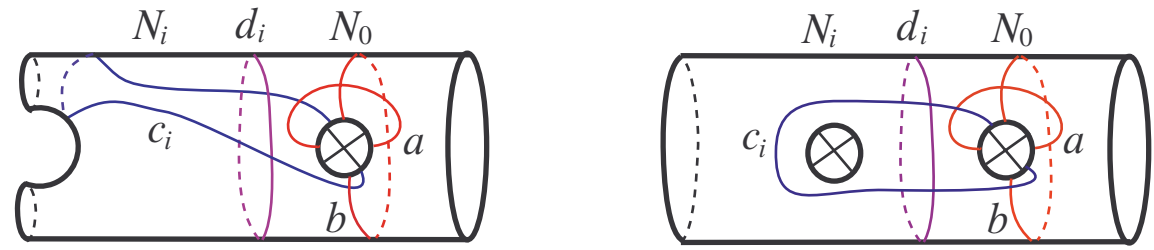

Figure 10. The circle $c_{i}$ if the genus of $N_{i}$ is zero or nonzero.

\subsection{More preparation}

Lemma 7.5. Let $\varphi$ be an automorphism of $C(N)$ and let $\alpha$ be an essential one-sided vertex in $C(N)$. If $\varphi$ fixes all vertices in $\operatorname{St}(\alpha)$, then it also fixes all vertices dual to $\alpha$.

Proof. Let $\beta$ be a vertex dual to $\alpha$. Let $a$ be a circle representing $\alpha$ and let $b$ be a circle representing $\beta$ such that $b$ intersects $a$ only once. Let $T$ be a regular neighborhood of $a \cup b$.

If $b$ is one-sided, then the surface $T$ is a real projective plane with two boundary components, $d_{1}$ and $d_{2}$. If one of $d_{i}$ is parallel to a boundary component, we just ignore it, and consider the other. Let $\delta_{1}$ and $\delta_{2}$ denote the isotopy classes of $d_{1}$ and $d_{2}$, respectively. Let $c$ be a representative of $\varphi(\beta)$ intersecting $d_{i}$ and $a$ minimally. Since $\delta_{i} \in L(\alpha)$, we have $\varphi\left(\delta_{i}\right)=\delta_{i}$. Since $\varphi(\beta)$ is connected by an edge to each $\delta_{i}$ and to the isotopy class of each circle disjoint from $T$, we conclude that $c$ lies on $T$. Up to isotopy, there are only two one-sided circles on $T ; a$ and $b$. Since $\beta \neq \alpha$, we have $\varphi(\beta) \neq \varphi(\alpha)=\alpha$, concluding that $c$ is isotopic to $b$. Thus we get $\varphi(\beta)=\beta$.

If $b$ is two-sided, then the surface $T$ is a Klein bottle with one boundary component, say $d$. Let $\delta$ denote the isotopy class of $d$. Let $c$ be a representative of $\varphi(\beta)$ intersecting $d$ and $a$ minimally. Since $\delta \in L(\alpha)$, we have $\varphi(\delta)=\delta$. Since $\varphi(\beta)$ is connected by an edge to $\delta$ and to the isotopy classes of circles disjoint from $T$, we conclude that $c$ lies on $T$. Since $c$ is two-sided and since up to isotopy there is only one two-sided nontrivial circle on a Klein bottle with one hole (cf. [19]), we conclude that $c$ is isotopic to $b$. Thus we get $\varphi(\beta)=\beta$.

Lemma 7.6. Let $\varphi$ be an automorphism of $C(N)$ and let $Y$ be a connected component of the graph $\tilde{X}(N)$. Suppose that $\varphi(\alpha)=\alpha$ whenever $\alpha$ is contained in $\operatorname{St}(\gamma) \cup D(\gamma)$ for some vertex $\gamma$ in $Y$. Then $\varphi$ is equal to the identity.

Proof. Note that, by Theorem 3.10, the graph $\tilde{X}(N)$ is either connected (if $g \neq 2$ or 3), or has two connected components (if $g=2$ ), or has three connected components (if $g=3$ ). Let $\alpha$ be a vertex of $C(N)$ represented by a circle $a$. In most cases, depending on the topological type of $\alpha$ we find a vertex $\beta$ in $Y$ such that $\alpha \in$ $\operatorname{St}(\beta) \cup D(\beta)$. In the following $\beta$ will denote the isotopy class of the circle $b$. 
Case 1. Suppose first that $g=1$ or $g \geq 4$, so that $Y=\tilde{X}(N)$. If $a$ is onesided and essential, then let $b=a$. If $a$ is one-sided but not essential (this occurs if $g \geq 4$ ), or if $a$ is two-sided and nonseparating, then let $b$ be any one-sided essential circle intersecting $a$ only once. In each of these cases, $\beta$ is a vertex of $\widetilde{X}(N)$ and $\alpha \in \operatorname{St}(\beta) \cup D(\beta)$, so that $\varphi(\alpha)=\alpha$.

Suppose that $a$ is separating. Then the surface $N_{a}$ has two connected components, $N_{1}$ and $N_{2}$. At least one of $N_{i}$, say $N_{1}$, is nonorientable. If $N_{1}$ and $N_{2}$ are both nonorientable, or the genus of $N_{1}$ is at least two, then let $b$ be any one-sided circle on $N_{1}$ such that its complement in $N$ is nonorientable. If $N_{2}$ is a sphere with holes and if the genus of $N$ is 1 , let $b$ be a one-sided circle on $N_{1}(b$ is essential since $g=1$ ). In all these cases, $\beta$ is a vertex in $\widetilde{X}(N)$, and $\alpha \in \operatorname{St}(\beta)$, so that $\varphi(\alpha)=\alpha$.

Suppose that the genus of $N_{1}$ is 1 and $N_{2}$ is orientable of positive genus (so that $g \geq 4$ and $g$ is odd). Let $P$ be a set consisting of a finite number of nonseparating circles on $N_{2}$ and a finite number of one-sided circles on $N_{1}$ such that any circle disjoint from $P$ is isotopic to $a$. Since $\varphi$ fixes the isotopy class of each element of $P$ and $\varphi(\alpha)$ is connected to each of them by an edge in $C(N)$, it follows that $\varphi(\alpha)=\alpha$.

Case 2. Suppose now that $g=2$. If $a$ is one-sided, then it is essential. In this case, either $\alpha$ is a vertex in $Y$, or there is a one-sided circle $c$ disjoint from $a$. Let $b=a$ in the first case, or $b=c$ in the second case. Hence, $\beta$ is a vertex $Y$ and $\alpha \in \operatorname{St}(\beta)$.

If $a$ is two-sided and nonseparating, then one can easily find two disjoint one-sided circles each of which intersects $a$ only once. The isotopy class, $\beta$, of one of these two circles is a vertex in $Y$. We then get $\alpha \in D(\beta)$.

Suppose that $a$ is two-sided and separating. Let $N_{1}$ and $N_{2}$ be the components of $N_{a}$. One of these components, say $N_{1}$, is nonorientable. If both $N_{1}$ and $N_{2}$ are of genus one, choose a one-sided circle on each component. The isotopy class, $\beta$, of one of these circles is a vertex in $Y$ and we have $\alpha \in \operatorname{St}(\beta)$. If $N_{1}$ is of genus two, let $b$ be any one-sided circle on $N_{1}$ whose isotopy class $\beta$ is a vertex in $Y$. Then we have $\alpha \in \operatorname{St}(\beta)$.

We conclude that if $g=2$ and if $\alpha$ is any vertex of $C(N)$, then $\varphi(\alpha)=\alpha$.

Case 3. Suppose finally that $g=3$. If $a$ is one-sided and essential, then there are two other one-sided essential circles $a_{1}$ and $a_{2}$ on $N$ such that $a, a_{1}$ and $a_{2}$ are pairwise disjoint. The isotopy class, $\beta$, of one of these three circles is a vertex in $Y$. Then we have $\alpha \in \operatorname{St}(\beta)$.

If $a$ is one-sided and but not essential, then there are three pairwise disjoint onesided essential circles intersecting $a$ only once. The isotopy class, $\beta$, of one of them must be a vertex in $Y$. Thus we have $\alpha \in D(\beta)$.

If $a$ is two-sided and nonseparating, then there is a vertex $\beta$ in $Y$ such that $\alpha \in D(\beta)$ or $\alpha \in L(\beta)$.

Suppose now that $a$ is two-sided and separating. Let $N_{1}$ and $N_{2}$ be the components of $N_{a}$. One of these components, say $N_{1}$, is nonorientable. If both $N_{i}$ are nonorientable, let $b$ denote a one-sided circle disjoint from $a$ such that its isotopy 
class $\beta$ is a vertex in $Y$. Then $\alpha \in \operatorname{St}(\beta)$.

If $N_{2}$ is a sphere with holes, then let $b$ denote a one-sided circle disjoint from $a$ such that its isotopy class $\beta$ is a vertex in $Y$. Then $\alpha \in \operatorname{St}(\beta)$.

It follows that $\varphi(\alpha)=\alpha$ in all above cases. The only remaining case is that $N_{1}$ is of genus 1 and $N_{2}$ is a torus with holes.

If $N_{2}$ is a torus with holes, let $P$ be a set consisting of a finite number of nonseparating circles on $N_{2}$ and a finite number of one-sided circles on $N_{1}$ such that any circle disjoint from $P$ is isotopic to $a$. Since the isotopy class of each element of $P$ is fixed by $\varphi$ and since $\varphi(\alpha)$ is connected to each of them by an edge, we must have $\varphi(\alpha)=\alpha$.

This completes the proof of the lemma.

7.4. Completing the proof of surjectivity. Finally, we are ready to prove the surjectivity of the natural homomorphism $\operatorname{Mod}(N) \rightarrow \operatorname{Aut} C(N)$. In the following theorem, it is convenient for us to consider the sphere as a nonorientable surface of genus zero.

Theorem 7.7. Let $N$ be a connected nonorientable surface of genus $g \geq 0$ with $n$ holes. Suppose that $g+n \geq 5$. Then the natural map $\Phi: \operatorname{Mod}(N) \rightarrow \operatorname{Aut} C(N)$ is surjective.

Proof. We prove the theorem by induction on $g$. If $g=0$ then $N$ is a sphere with at least 5 holes. By Theorem 3, the homomorphism $\Phi$ is surjective. Suppose that $N$ is a nonorientable surface of genus $g \geq 1$ and that the theorem holds for nonorientable surfaces of genus $g-1$. Let $\varphi: C(N) \rightarrow C(N)$ be a simplicial automorphism. We will prove that there is a mapping class $h$ on $N$ such that $\varphi(\alpha)=h(\alpha)$ for all vertices $\alpha$ of $C(N)$. For a mapping class $h$ we denote the automorphism $\Phi(h)$ of $C(N)$ also by $h$.

Let $\alpha$ be a vertex in $C(N)$ represented by a one-sided essential circle $a$, so that the surface $N_{a}$ obtained by cutting $N$ along $a$ is a nonorientable surface of genus $g-1$ with $n+1$ holes. By Theorem 5.1, there is a mapping class $f_{\alpha}$ such that $\varphi(\alpha)=f_{\alpha}(\alpha)$. Let $\varphi_{\alpha}=f_{\alpha}^{-1} \circ \varphi$, so that we have $\varphi_{\alpha}(\alpha)=\alpha$.

Now, $\varphi_{\alpha}$ restricts to an automorphism $\varphi_{\alpha}: L(\alpha) \rightarrow L(\alpha)$. Since $L(\alpha)$ is isomorphic to the complex of curves $C\left(N_{a}\right)$, we have an automorphism $\varphi_{\alpha}$ of the complex of curves of $N_{a}$. By induction, $\varphi_{\alpha}$ is equal to a map $\bar{g}_{\alpha}: C\left(N_{a}\right) \rightarrow C\left(N_{a}\right)$ which is induced by a diffeomorphism $\bar{G}_{\alpha}: N_{a} \rightarrow N_{a}$. Let $\partial_{a}$ be the boundary component of $N_{a}$ coming from $a$. Let us identify $\partial_{a}$ with the unit circle so that antipodal points on $\partial_{a}$ map to the same point of $a$ under the identification map $N_{a} \rightarrow N$.

We claim that $\bar{G}_{\alpha}\left(\partial_{a}\right)=\partial_{a}$. If $n=0$ then $\partial_{a}$ is the only boundary component of $N_{a}$. Thus there is nothing to show in this case. Suppose that $n \geq 2$. Let $\partial_{1}$ be a boundary component of $N$. Choose another boundary component $\partial_{2}$, and a circle $c$ disjoint from $a$ such that $c$ bounds a disk with two boundary components, $\partial_{1}$ and $\partial_{2}$. Let $\gamma$ denote the isotopy class of $c$. Then any circle $c^{\prime}$ representing $\varphi(\gamma)=\bar{g}_{\alpha}(\gamma)$ 
bounds a disk with two boundary components $\partial_{1}^{\prime}$ and $\partial_{2}^{\prime}$ of $N$ (and of $N_{a}$ ). Note that $\left\{\bar{G}_{\alpha}\left(\partial_{1}\right), \bar{G}_{\alpha}\left(\partial_{2}\right)\right\}=\left\{\partial_{1}^{\prime}, \partial_{2}^{\prime}\right\}$. It follows that $\bar{G}_{\alpha}$ permutes the boundary components of $N_{a}$ that are also boundary components on $N$, and hence $\bar{G}_{\alpha}\left(\partial_{a}\right)=\partial_{a}$. If $n=1$ then by taking $c$ as a circle bounding a Klein bottle $K$ containing $a$ such that $c$ is disjoint from $a$ one can show that $\bar{G}_{\alpha}\left(\partial_{a}\right)=\partial_{a}$.

By composing $\bar{G}_{\alpha}$ with a diffeomorphism isotopic to identity, we can assume that $\bar{G}_{\alpha}$ maps antipodal points on the boundary $\partial_{a}$ to antipodal points. Thus, $\bar{G}_{\alpha}$ induces a diffeomorphism $G_{\alpha}: N \rightarrow N$, which satisfies $G_{\alpha}(a)=a$. If $g_{\alpha}$ denote the isotopy class of $G_{\alpha}$, then $\varphi_{\alpha}$ agrees with $g_{\alpha}$ on every vertex of $\operatorname{St}(\alpha)$. The composition $g_{\alpha}^{-1} \circ \varphi_{\alpha}$ fixes every vertex of $\operatorname{St}(\alpha)$. By Lemma 7.5, $\varphi_{\alpha}$ fixes every vertex of $\operatorname{St}(\alpha) \cup D(\alpha)$.

Let $h_{\alpha}=f_{\alpha} \circ g_{\alpha}$, so that $\varphi(\gamma)=h_{\alpha}(\gamma)$ for all $\gamma \in \operatorname{St}(\alpha) \cup D(\alpha)$. That is, for each one-sided essential vertex $\alpha$, there is a mapping class $h_{\alpha}$ such that

$$
\varphi=h_{\alpha} \text { on } \operatorname{St}(\alpha) \cup D(\alpha) \text {. }
$$

Let $\beta$ be a one-sided essential vertex connected to $\alpha$ by an edge in the graph $\tilde{X}(N)$. We then have $h_{\alpha}(\gamma)=\varphi(\gamma)=h_{\beta}(\gamma)$ for all vertices $\gamma$ in $(\operatorname{St}(\alpha) \cup D(\alpha)) \cap$ $(\operatorname{St}(\beta) \cup D(\beta))$. By Theorem 7.4, we have $h_{\alpha}=h_{\beta}$.

It follows that if $\alpha$ and $\beta$ are two vertices in the same connected component of $\tilde{X}(N)$, then $h_{\alpha}=h_{\beta}$.

Fix an essential one-sided vertex $\alpha$ in $\tilde{X}(N)$ and let $h$ denote the mapping class $h_{\alpha}$. Let $Y$ be the component of $\widetilde{X}(N)$ containing $\alpha$. If $\beta$ is a vertex of $C(N)$ contained in $\operatorname{St}(\gamma) \cup D(\gamma)$ for some vertex $\gamma$ of $Y$, then $\varphi(\beta)=h_{\gamma}(\beta)=h(\beta)$. By Lemma 7.6, $\varphi(\gamma)=h(\gamma)$ for all vertices of $C(N)$.

This concludes the proof of the theorem.

\section{Exceptional cases}

In this last section, we look at the cases not covered by Theorem 1. Let $\Phi: \operatorname{Mod}(N) \rightarrow$ Aut $C(N)$ denote the natural homomorphism.

If $(g, n)=(1,0)$ then $\operatorname{Mod}(N)$ is trivial and the complex $C(N)$ consists of only one vertex, so that the map $\Phi$ is an isomorphism.

If $(g, n)=(1,1)$ then $\operatorname{Mod}(N)$ is (isomorphic to) the cyclic group $\mathbb{Z}_{2}$ of order two and $C(N)$ consists of one vertex. Hence, $\Phi$ is onto, but not one-to-one.

If $(g, n)=(1,2)$ then $\operatorname{Mod}(N)$ is the dihedral group of order eight ([14], Corollary 4.6). The complex $C(N)$ consists of two vertices, so that its automorphism group is $\mathbb{Z}_{2}$. It follows from Theorem 2.4 that $\Phi$ is onto and its kernel is $\mathbb{Z}_{2} \oplus \mathbb{Z}_{2}$, generated by $v_{1} v_{2}$ and the braid interchanging two boundary components.

If $(g, n)=(2,0)$ then $\operatorname{Mod}(N)$ is $\mathbb{Z}_{2} \oplus \mathbb{Z}_{2}$. The complex $C(N)$ consists of three vertices and one edge joining two one-sided vertices (see Section 2.4). The Dehn twist about the unique two-sided nontrivial circle acts nontrivially on $C(N)$. Hence, the map $\Phi$ is onto and its kernel is $\mathbb{Z}_{2}$. 
If $(g, n)=(2,1)$ then $C(N)$ is the disjoint union of a vertex (the isotopy class of the unique two-sided circle $a$ ) and the real line $\ell$ on which a vertex is placed at each integer point ([19]), so that Aut $C(N)$ is an infinite dihedral group. The vertex set on $\ell$ is $\left\{t_{a}^{k}(\beta): k \in \mathbb{Z}\right\}$, and $t_{a}^{k}(\beta)$ is connected to $t_{a}^{k+1}(\beta)$ for each $k$. Here, $\beta$ is the isotopy class of any fixed one-sided circle. Thus, the Dehn twist $t_{a}$ acts as a translation on $\ell$. Clearly, there is a mapping class acting as the reflection on $\ell$. Thus, the map $\Phi$ is onto. One can easily to find a nontrivial diffeomorphism of $N$ acting trivially on $C(N)$, so that $\Phi$ is not one-to-one.

Suppose now that $(g, n)=(3,0)$. Let $T$ be a torus with one boundary component $\partial$. Assume that $N$ is obtained from $T$ by identifying the antipodal points on $\partial$. Every diffeomorphism $T \rightarrow T$ is isotopic to a diffeomorphism which maps antipodal points to antipodal points on $\partial$, so that it induces a diffeomorphism $N \rightarrow N$. Identifying the extended mapping class group of $T$ by $G L(2, \mathbb{Z})$ gives a homomorphism $\psi: G L(2, \mathbb{Z}) \rightarrow \operatorname{Mod}(N)$. It is easy to see that $\psi$ is injective. Since the image $d$ in $N$ of $\partial$ is the unique (up to isotopy) one-sided circle whose complement is orientable, the map $\psi$ is also surjective, so that it is an isomorphism. The image of the hyperelliptic involution of $T$ acts trivially on the curve complex, in fact it is the only such element. Thus, the kernel of the map $\Phi: \operatorname{Mod}(N) \rightarrow \operatorname{Aut} C(N)$ is isomorphic to $\mathbb{Z}_{2}$.

Let us denote by $\delta$ the isotopy class of $d$. Since any circle whose complement in $N$ is orientable is isotopic to $d$, the vertex $\delta$ is fixed by all automorphisms of $C(N)$. Note that all two-sided vertices are connected to $\delta$, so that the link $L(\delta)$ of $\delta$ is naturally isomorphic to the curve complex $C(T)$. Also, given a two-sided vertex $\alpha$ there exists a unique (one-sided) essential vertex $\alpha^{\prime}$ connected to $\alpha$. It can be seen that for any two two-sided vertices $\alpha$ and $\beta, i(\alpha, \beta)=1$ if and only if the corresponding essential vertices $\alpha^{\prime}$ and $\beta^{\prime}$ are connected in $C(N)$. Since an automorphism of the curve complex of the torus $T$ preserving the geometric intersection one property is induced by a diffeomorphism of $T$, it follows that all automorphisms of $C(N)$ are induced by diffeomorphisms of $N$. That is, the map $\Phi$ is onto.

In the remaining four exceptional cases, the cases where $g+n=4$, we do not know whether the natural map $\operatorname{Mod}(N) \rightarrow \operatorname{Aut} C(N)$ is an isomorphism.

\section{References}

[1] F. Atalan, Automorphisms of complex of curves on odd genus nonorientable surfaces. $\mathrm{Ph} . \mathrm{D}$. thesis, Middle East Technical University, 2005.

[2] R. W. Bell and D. Margalit, Injections of Artin groups. Comment. Math. Helv. 82 (2007), 725-751. Zbl 1148.20024 MR 2341838

[3] T. E. Brendle and D. Margalit, Commensurations of the Johnson kernel. Geom. Topol. 8 (2004), 1361-1384. Zbl 1079.57017 MR 2119299

[4] W. J. Harvey, Boundary structure of the modular group. In Riemann surfaces and related topics: Proceedings of the 1978 Stony Brook Conference (State Univ. New York, Stony 
Brook, N.Y., 1978), Ann. of Math. Stud. 97, Princeton Univ. Press, Princeton, N.J., 1981, 245-251. Zbl 0461.30036 MR 624817

[5] E. Irmak, Superinjective simplicial maps of complexes of curves and injective homomorphisms of subgroups of mapping class groups. Topology 43 (2004), 513-541. Zbl 1052.57024 MR 2041629

[6] E. Irmak, Superinjective simplicial maps of complexes of curves and injective homomorphisms of subgroups of mapping class groups. II. Topology Appl. 153 (2006), 1309-1340. Zbl 1100.57020 MR 2202856

[7] E. Irmak, Complexes of nonseparating curves and mapping class groups. Michigan Math. J. 54 (2006), 81-110. Zbl 1131.57019 MR 2214789

[8] E. Irmak, Superinjective simplicial maps of the complexes of curves on nonorientable surfaces. Turkish J. Math. 36 (2012), 407-421. Zbl 1251.32013 MR 2993573

[9] E. Irmak and M. Korkmaz, Automorphisms of the Hatcher-Thurston complex. Israel J. Math. 162 (2007), 183-196. Zbl 1149.57032 MR 2365859

[10] N. V. Ivanov, Automorphisms of Teichmüller modular groups. In Topology and geometry - Rohlin Seminar, Lecture Notes in Math. 1346, Springer, Berlin 1988, 199-270. Zbl 0657.57004 MR 970079

[11] N. V. Ivanov, Automorphism of complexes of curves and of Teichmüller spaces. Internat. Math. Res. Notices 1997 (1997), 651-666. Zbl 0890.57018 MR 1460387

[12] N. V. Ivanov, Isometries of Teichmüller spaces from the point of view of Mostow rigidity. In Topology, ergodic theory, real algebraic geometry, Amer. Math. Soc. Transl. Ser. (2) 202, Amer. Math. Soc., Providence, RI, 2001, 131-149. Zbl 1113.30305 MR 1819186

[13] M. Korkmaz, Automorphisms of complexes of curves on punctured spheres and on punctured tori. Topology Appl. 95 (1999), 85-111. Zbl 0926.57012 MR 1696431

[14] M. Korkmaz, Mapping class groups of nonorientable surfaces. Geom. Dedicata 89 (2002), 109-133. Zbl 1016.57013 MR 1890954

[15] F. Luo, Automorphisms of the complex of curves. Topology 39 (2000), 283-298. Zbl 0951.32012 MR 1722024

[16] D. Margalit, Automorphisms of the pants complex. Duke Math. J. 121 (2004), 457-479. Zbl 1055.57024 MR 2040283

[17] J. J. Rotman, An introduction to algebraic topology. Graduate Texts in Mathematics 119, Springer-Verlag, New York 1988. Zbl 0661.55001 MR 957919

[18] S. Schleimer, Notes on the complex of curves. Caltech, minicourse Jan. 2005 (revised 11/23/2006). http://homepages.warwick.ac.uk/ masgar/Maths/notes.pdf

[19] M. Scharlemann, The complex of curves on non-orientable surfaces. J. London Math. Soc. (2) 25 (1982), 171-184. Zbl 0479.57005 MR 645874

[20] P. Schmutz Schaller, Mapping class groups of hyperbolic surfaces and automorphism groups of graphs. Compositio Math. 122 (2000), 243-260. Zbl 0981.57004 MR 1781329

[21] K. J. Shackleton, Combinatorial rigidity in curve complexes and mapping class groups. Pacific J. Math. 230 (2007), 217-232. Zbl 1165.57017 MR 2318453 
Received July 15, 2012; revised October 11, 2012

F. Atalan, Department of Mathematics, Atilim University, 06836 Ankara, Turkey

E-mail: fatalan@atilim.edu.tr

M. Korkmaz, Department of Mathematics, Middle East Technical University, 06800 Ankara, Turkey, and Max-Planck-Institut für Mathematik, Vivatsgasse 7, 53111 Bonn, Germany

E-mail: korkmaz@metu.edu.tr 